\title{
Self-diffusion coefficients of methane/n-hexane mixtures at high pressures: an evaluation of the finite-size effect and a comparison of force fields
}

\author{
Thiago J. P. dos Santos ${ }^{\mathrm{a}}$, Charlles R. A. Abreu ${ }^{\mathrm{a}, *}$, Bruno A. C. Horta ${ }^{\mathrm{b}}$, \\ Frederico W. Tavares ${ }^{\mathrm{a}, \mathrm{c}}$ \\ ${ }^{a}$ School of Chemistry, Federal University of Rio de Janeiro, Rio de Janeiro 68542, Brazil \\ ${ }^{b}$ Institute of Chemistry, Federal University of Rio de Janeiro, Rio de Janeiro 21941-909, \\ Brazil \\ ${ }^{c}$ Chemical Engineering Program - COPPE, Federal University of Rio de Janeiro, Rio de \\ Janeiro 68542, Brazil
}

\begin{abstract}
Mass transport coefficients play an important role in process design and in compositional grading of oil reservoirs. As experimental measurements of these properties can be costly and hazardous, Molecular Dynamics simulations emerge as an alternative approach. In this work, we used Molecular Dynamics to calculate the self-diffusion coefficients of methane/n-hexane mixtures at different conditions, in both liquid and supercritical phases. We evaluated how the finite box size and the choice of the force field affect the calculated properties at high pressures. Results show a strong dependency between self-diffusion and the simulation box size. The Yeh-Hummer analytical correction [J. Phys. Chem. B, 108,15873 (2004)] can attenuate this effect, but sometimes makes the results depart from experimental data due to issues concerning the force fields. We have also found that different all-atom and united-atom models can produce biased results due to caging effects and to different dihedral configurations of the n-alkane.
\end{abstract}

Keywords: Self-diffusion, Molecular Dynamics Simulations, n-alkanes, Force Fields

\footnotetext{
${ }^{*}$ Corresponding author

Email address: abreu@eq.ufrj.br (Charlles R. A. Abreu )
}

Preprint submitted to The Journal of Supercritical Fluids

July 31, 2019 


\section{Introduction}

The determination of mass transport properties has always been indispensable to the design of industrial processes and to chemical engineering as a whole 1. Among its many applications, mass transport coefficients are important 5 for separation and extraction processes, multiphase reactions, flow in porous media, and for several operations in the oil and gas industry [2, 3, 4]. Mass transport coefficients are also quite important for reservoir engineering because the compositional grading of oil reservoirs involves a good interpretation on the self-diffusion, mutual diffusion, and thermodiffusion processes at high pressures

10 [5, 6. In particular, the self-diffusion coefficient is useful for describing the structure and the thermophysical behavior of fluids [7. Unlike ordinary or mutual diffusion, the self-diffusion phenomenon occurs in the absence of a chemical potential gradient, when molecules collide with each other and diffuse simply due to Brownian motion [8, 9, 10,

Experimentally, self-diffusion coefficients for both pure substances and mixtures are commonly determined through the well established pulsed field gradient NMR technique [11, 12, 13. However, despite the need for transport coefficient data under reservoir conditions [13, experimental measurements for hydrocarbon systems at high pressures always present an inherent safety risk and high cost. In this context, Molecular Dynamics (MD) simulation can be used to study the self-diffusive process of high-pressure systems [14, 15] without the mentioned problems of experimental measurements.

There are two common approaches to calculate self-diffusion coefficients using MD simulations. The first one is based on the evaluation of the mean square displacement (MSD) of the molecules over time, whose slope is a constant directly proportional to the self-diffusion coefficient [8, 16]. This is commonly known as the Einstein relation [17, from which the self-diffusion coefficient of a component $i$ can be computed as

$$
D_{i}=\lim _{t \rightarrow \infty} \frac{1}{6 t}\left\{\frac{1}{N_{i}} \sum_{j=1}^{N_{i}}\left[\boldsymbol{r}_{\boldsymbol{j}}(t)-\boldsymbol{r}_{\boldsymbol{j}}(0)\right]^{2}\right\},
$$


where $t$ is the time, $N_{i}$ is the number of molecules of component $i$, and $\boldsymbol{r}_{\boldsymbol{j}}$ is the center-of-mass coordinate of a molecule $j$ of this component. The term inside curly brackets is the mean square displacement (MSD).

The second approach to obtain self-diffusion coefficients via Molecular Dynamics relies on the so-called Green-Kubo relations [18, 19]. The self-diffusion coefficient depends intrinsically on the velocity autocorrelation function of individual particles [20], and this relation can be expressed by

$$
D_{i}=\frac{1}{3 N_{i}} \sum_{j=1}^{N_{i}} \int_{0}^{\infty}\left[\boldsymbol{v}_{\boldsymbol{j}}(t) \cdot \boldsymbol{v}_{\boldsymbol{j}}(0)\right] d t,
$$

where $\boldsymbol{v}_{\boldsymbol{j}}$ is the velocity vector of each molecule $j$ of type $i$.

Both approaches consider the self-diffusion coefficient as an average along the three independent directions $x, y$, and $z$, with the hypothesis of an isotropic system. In theory, both Equations (1) and (2) are equivalent and should lead to the same self-diffusion coefficient value 21. In practice, it has been shown that the two approaches lead to very similar results for simple systems such as Lennard-Jones fluids 1, 22. However, most authors prefer to use the Einstein relation due to its robustness [23, 24] and to some other advantages. Namely, (i) it is not necessary to consider integration limits, (ii) the MSD presents less 35 noise than self-correlation functions, (iii) the coefficient can be calculated by a simple linear adjustment, and (iv) deviations from linearity can be physically interpreted (e.g. ballistic effects). In both approaches, the results can be statistically enhanced by using averages calculated over multiple time origins [25].

It is important to note, however, that MD simulations are not immune to problems and may also present certain limitations that hinder the proper calculation of transport properties. It has been pointed out that the finite size of the simulation box can lead to an underestimation of the calculated selfdiffusion coefficient, since long-range hydrodynamic interactions are not properly accounted for [7, 26, 27]. In 1993, Dünweg and Kremer [28] introduced a correction term for finite-size effects on the calculation of self-diffusivity based on the Kirkwood-Riseman theory. The main idea was to determine the hydrodynamic self-interactions by comparing modified Oseen tensors for the case 
of a finite and periodic system and for the case of an infinite and nonperiodic system. More than two decades later, Yeh and Hummer [29] showed that disregarded long-range hydrodynamic interactions can lead to underestimation of the self-diffusion coefficient even for small molecules like water, resulting in a systematic error of about $10 \%$ for systems with 2000 water molecules in a periodic cubic cell. Then, they derived an analytical correction factor by modeling the hydrodynamics of a particle in a viscous and periodic cubic system, which led to the same correction provided by Dünweg and Kremer [28, given by

$$
D_{i, \infty}=D_{i}+\frac{\zeta k_{b} T}{6 \pi \eta L}
$$

where $D_{i, \infty}$ is the self-diffusion coefficient in the thermodynamic limit, $D_{i}$ is

40 the value obtained via MD simulations and either Equation (1) or (2) (therefore liable to finite-size effects), $k_{b}$ is the Boltzmann constant, $T$ is the absolute temperature, $L$ is the cell length, $\eta$ is the kinematic viscosity, and $\zeta \approx 2.837297$ [30, 31. Many authors have already discussed about the importance of the finitesize effects on the calculation of self-diffusivity and the improvement provided by the Yeh-Hummer correction factor for some varied set of cases, such as LennardJones fluids, hard-sphere fluids, and systems containing poly(ethylene glycol) dimethyl ethers, pure n-alkanes, water, $\mathrm{CO}_{2}, \mathrm{HCO}_{3}{ }^{-}$and $\mathrm{CO}_{3}{ }^{2-}$ [7, 32, 33, 34, 23 .

In 2007, an empirical correction factor was proposed for systems with complex density dependence, which converges to the Yeh-Hummer correction factor for most cases [35. Despite all this, correction factors have been frequently neglected and compensated by the use of large simulation boxes or large cut-off distances [36, in an unsystematic and unpredictable way. Recently, Gonçalves et al. (2019) 37] evaluated the effect of the Lennard-Jones cut-off radius $\left(r_{c}\right)$ on 55 the calculation of thermodynamic and transport properties, showing that there is great sensitivity in the case of non-polar substances. Therefore, it is essential that the original cut-off recommendation of each molecular model be respected, since this is also a force-field parameter.

Another problem is that some force fields are already parameterized to target 
transport properties and implicitly bypass the finite-size effects by less appropriate means [38. In such cases, if due regard is not given to the force field, the use of the Yeh and Hummer correction factor may worsen the accuracy of the results compared to experimental data. Some other force fields may simply overestimate the self-diffusion coefficient under certain conditions [39] (not underestimate, as commonly expected due to finite-size effect), and once again the use of this positive correction factor should make the results apparently less accurate. Most of the commonly used hydrocarbon force fields, such as the OPLS [40, 41] and GROMOS [42, 43, 44] families, as well as NERD 45] and TraPPE [46], are parameterized so as to reproduce thermodynamic and phase equilibrium properties. Generally, a well-calibrated force field that reproduces a reasonable set of thermodynamic properties also tends to reproduce transport properties satisfactorily [47, even without the finite-size correction factor. Of course, there are several aspects of these force fields that may, by chance, mask the underestimation caused by the finite-size effect and provide self-diffusivity results close to experimental data. The main problem is that, although the finite size effect does not seem to have much relevance in these cases, the dimensions of the simulation box can still greatly influence the results. Thus, it seems to be inappropriate to solve an inherent limitation caused by the size of the simulation box by compensating it in other aspects of the force field without the proper 80 phenomenological treatment of the problem.

In this work, we have calculated the self-diffusion coefficients of several methane/n-hexane mixtures at reservoir conditions using MD simulations. We systematically evaluated three different united-atom and one all-atom force fields in terms of diffusion coefficients calculated at high pressure conditions. We also investigated the finite-size effects of the simulation box in the calculation of the self-diffusivity. We also verified the efficacy of the Yeh-Hummer correction factor at high pressures and the occurrence of issues such as a caging effect and the relation between the dihedral-angle distribution and self-diffusivity of molecules, for all tested force fields in both liquid and supercritical phases. 


\section{Simulation Details}

We have studied several methane(1)/n-hexane(2) mixtures under reservoir conditions, with different compositions $\left(x_{1}=0.27 ; 0.48 ; 0.52 ; 0.72\right)$ under pressures of 30,40 and $50 \mathrm{MPa}$, and at temperatures of 303.2 and $333.1 \mathrm{~K}$. The simulation boxes were built with the software Playmol [48], and simulations were performed using the LAMMPS package [49]. We evaluated different force fields (FF) for the hydrocarbons, namely NERD (united atoms) [45], OPLS (united atoms) [40, OPLS (all-atoms) [4], and GROMOS (united atoms) 43, 42] with the quartic bond potential rather than the rigid bonds. All force fields were used exactly in accordance with their original parameters and recommendations.

The size of all systems was fixed be setting $\mathrm{N}=1000$ molecules placed in a cubic simulation box with periodic boundary conditions. Simulations were performed using a 1 fs timestep and, in the case of the all-atom force field, the long-range interactions were calculated by the PPPM method [50, considering an accuracy of 0.0001 for the relative error in forces. Temperature and pressure were controlled by the use of Nosé-Hoover chain thermostats [51] and the Martyna-Tobias-Klein barostat [52, with their characteristic time scales set to $100 \mathrm{fs}$ and $1000 \mathrm{fs}$, respectively. The equations of motion were integrated by using the LAMMPS implementation of the method described by Tuckerman et al. (2006) 53.

Initially, the simulation boxes were built with arbitrary densities. The densities were then equilibrated by performing $7 \mathrm{~ns}$ of NpT simulations. New simulation boxes were built with the computed average densities and were subjected to 1 ns equilibration in the canonical ensemble (NVT), followed by a 5 ns production in the same ensemble. All simulations were performed in triplicate to ensure statistical reliability. During the data production step, the center-ofmass positions of all molecules in the system were stored at every $200 \mathrm{fs}$. The stress tensor components $\tau_{x y}, \tau_{x z}$ and $\tau_{y z}$ were stored at every $10 \mathrm{fs}$.

We calculated the self-diffusion coefficients of each component using the Einstein relation (Equation (1)). We also determined the self-diffusion coefficients 
corrected by the Yeh-Hummer correction factor using Equation (3). The kinematic viscosity $\eta$, which is necessary for calculating the Yeh-Hummer correction factor, was calculated by the corresponding Green-Kubo relation

$$
\eta=\int_{0}^{\infty}\left\langle\tau_{\alpha \beta}(t) \cdot \tau_{\alpha \alpha}(0)\right\rangle d t
$$

We do not take into account the finite-size effects on the viscosity, since this effect is small and has no clear dependency on the size of the simulation box [7. To increase the statistical quality of the results, we employed an algorithm of multiple origins in time to determine both the self-diffusion coefficient and the viscosity [25].

For all simulated systems, we compared our results to experimental data by calculating the Absolute Average Deviation (AAD), given by the expression

$$
A A D=\frac{1}{N_{s}} \sum_{s=1}^{N_{s}}\left|D_{i}-D_{i, E x p}\right|,
$$

where $N_{s}$ is the number of samples, $D_{i}$ is the self-diffusion coefficient obtained by Molecular Dynamics simulations, and $D_{i, E x p}$ is its experimental value. We also performed the calculation of the Average Deviation (AD) for groups of samples with the same deviation signal, in order to evaluate the mean deviation signal behavior without taking biased averages by error compensations. The $\mathrm{AD}$ is similar to the AAD, but involves the actual value of the difference between $D_{i}$ and $D_{i, E x p}$, that is,

$$
A D=\frac{1}{N_{s}} \sum_{s=1}^{N_{s}}\left(D_{i}-D_{i, \operatorname{Exp}}\right) .
$$

\section{Results and discussion}

\subsection{Density and viscosity results}

The results of density and viscosity for all mixtures and force fields are listed in Tables S1 S4 of the Supplementary Material (4). The obtained density results showed excellent agreement with the values predicted by the GERG2008 equation of state [54]. Viscosity results also presented good agreement 
with the predictions of the REFPROP software [55], which calculates viscosity using fluid-specific correlations, the ECS method, or the friction theory method.

According to the estimates of critical coordinates also provided by REFPROP, under the studied conditions all mixtures with methane(1) fractions $x_{1}=0.27,0.52$, and 0.72 are found in the compressible-liquid phase region, while all mixtures with $x_{1}=0.88$ are in the supercritical phase region. All the studied systems were in homogeneous phase and in reservoir conditions near the critical transition.

\subsection{Self-diffusion and finite-size effect}

The self-diffusion coefficients for both components in all mixtures and for all evaluated force fields are shown in Tables $\widehat{S 5}$ |S12 of the Supplementary Masystem density, thus enhancing molecular diffusion. Finally, as can be seen from the result tables in the supplementary material, as temperature rises the density 
decreases and molecular diffusion is enhanced.

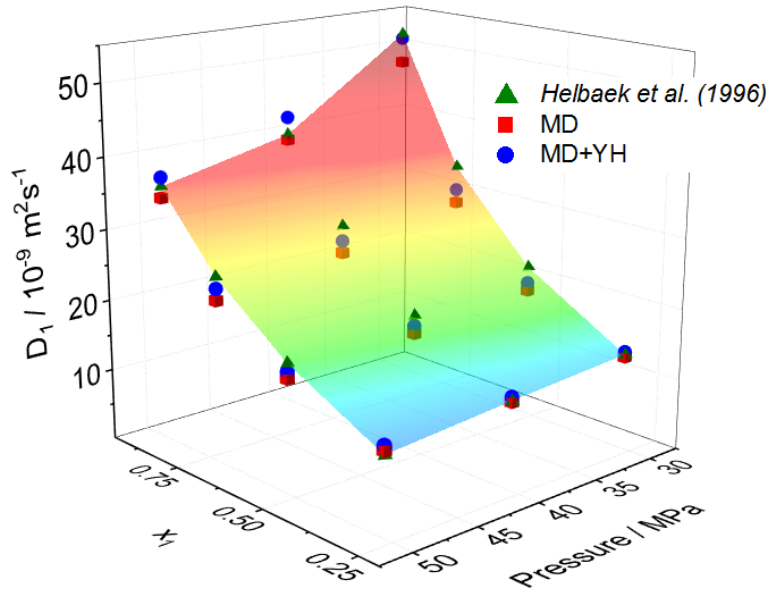

Figure 1: Self-diffusion coefficients of methane(1) in methane(1)/n-hexane(2) mixtures at $333.1 \mathrm{~K}$, obtained with OPLS-UA Force Field. The surface was obtained by fitting the experimental data. $\triangle$ Experimental data; $\square$ Molecular Dynamics; $\bigcirc$ Molecular Dynamics with the Yeh-Hummer correction.

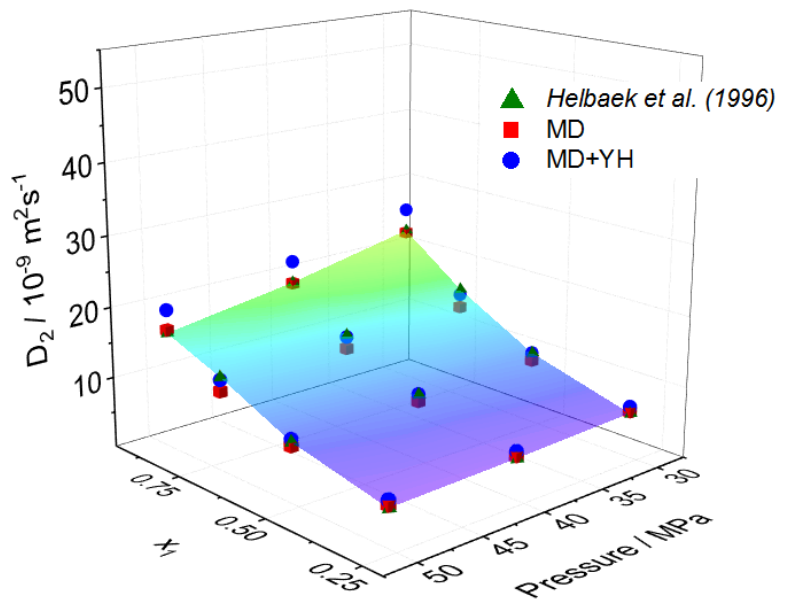

Figure 2: Self-diffusion coefficients of $n$-hexane(2) in methane(1)/n-hexane(2) mixtures at $333.1 \mathrm{~K}$, obtained with OPLS-UA Force Field. The surface was obtained by fitting the experimental data. $\triangle$ Experimental data; $\square$ Molecular Dynamics; $\bigcirc$ Molecular Dynamics with the Yeh-Hummer correction. 
It is important to mention that the Yeh-Hummer correction factor showed excellent consistency in the prediction of the self-diffusion coefficients freed from finite size effects. Figures 3 and 4 show how the Yeh-Hummer factor corrects the long-range hydrodynamic interactions and eliminates the dependence on system size of the self-diffusivity of both methane(1) and n-hexane(2), with $x_{1}=0.72$, at $40 \mathrm{MPa}$, and $333.1 \mathrm{~K}$. Moreover, the Yeh-Hummer correction factor seems to have solved the problem of finite-size effect regardless of the thermodynamic condition or the force field employed. Similar results showing the finite-size effect on self-diffusion coefficients and the impact of the Yeh-Hummer correction factor for pure systems and for other mixtures have already been reported in the literature [7, 29].

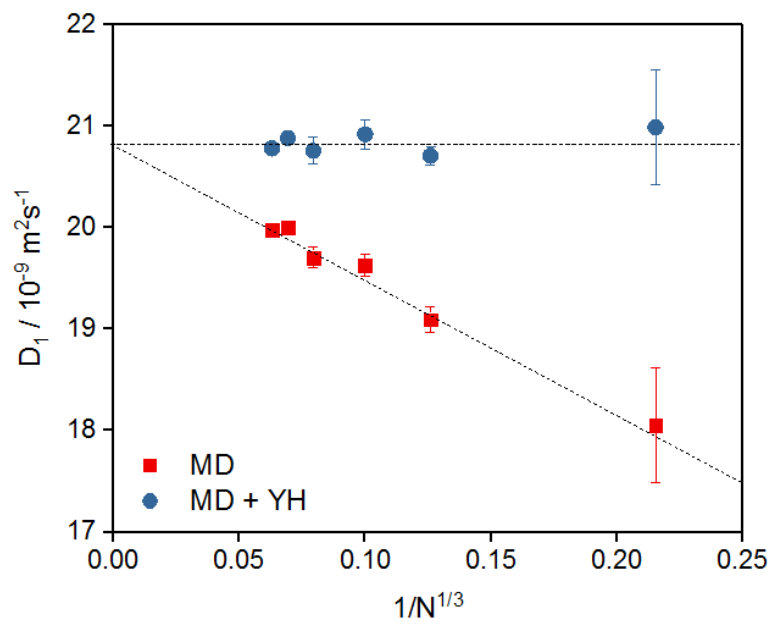

Figure 3: Self-diffusion coefficients of methane(1) in different simulation box sizes obtained with OPLS-UA, for mixture with $x_{1}=0.72, \mathrm{P}=40 \mathrm{MPa}$ and $\mathrm{T}=333.1 \mathrm{~K}$. $\square$ Molecular Dynamics; $\bigcirc$ Molecular Dynamics with the Yeh-Hummer correction. 


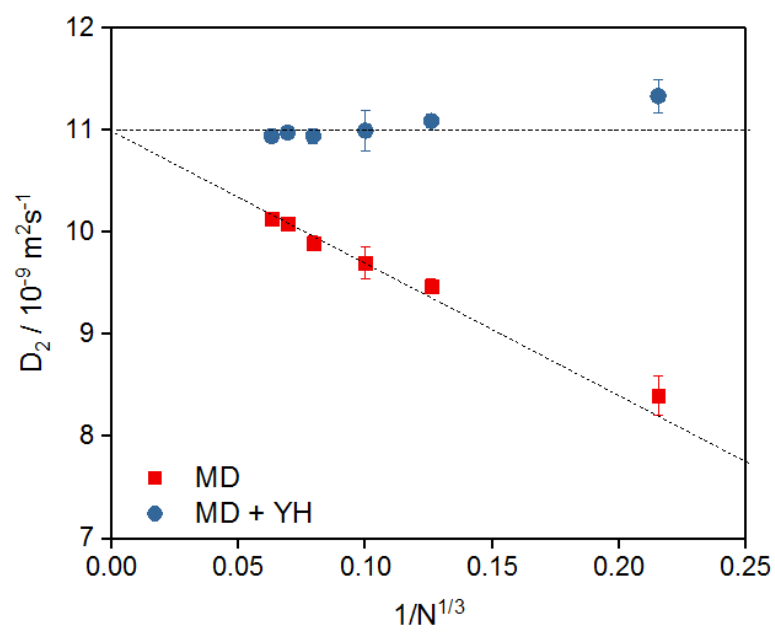

Figure 4: Self-diffusion coefficients of n-hexane(2) in different simulation box sizes obtained with OPLS-UA, for mixture with $x_{1}=0.72, \mathrm{P}=40 \mathrm{MPa}$ and $\mathrm{T}=333.1 \mathrm{~K}$. $\square$ Molecular Dynamics; $\bigcirc$ Molecular Dynamics with the Yeh-Hummer correction.

In order to systematically compare the self-diffusion coefficients obtained with different force fields and to evaluate the improvement provided by the Yeh-Hummer correction in each one of them, we analyzed the Absolute Average Deviation (AAD) computed along isotherms for both components of the mixture, taking averages over all simulated compositions and pressures. Tables 1 and 2 contain the compiled AAD results for the self-diffusion coefficients of methane and n-hexane, respectively, in the two simulated isotherms. In general, all force fields showed deviations from the experimental data within an acceptable range. In most cases, the use of the Yeh-Hummer correction brought the self-diffusion coefficients closer to experimental data, reducing the absolute average deviation of the results. However, although it works well on average, there are some cases in which the Yeh-Hummer correction factor moves the results away from the experimental data and increases the AAD when compared to the uncorrected results. Table 2 shows that, at $\mathrm{T}=333.1 \mathrm{~K}$, the use of the 185 Yeh-Hummer correction factor tends to make the self-diffusion coefficients depart from the experimental results. It is worth noting that the uncorrected 
values are already quite close to the experimental data and the addition of the correction, which is always positive, leads to overestimation.

Table 1: Comparison of the AAD on the isotherms for the self-diffusivity of methane(1) without (MD) and with the Yeh-Hummer correction (MD+HY), for different force fields.

AAD is calculated over four compositions and three pressures.

\begin{tabular}{lcc|cc|cc}
\cline { 2 - 7 } & \multicolumn{5}{c}{$D_{1} A A D / 10^{-9} m^{2} s^{-1}$} \\
\cline { 2 - 7 } & \multicolumn{2}{c}{$\mathbf{T}=\mathbf{3 0 3 . 2} \mathbf{~ K}$} & \multicolumn{2}{c}{$\mathbf{T}=\mathbf{3 3 3 . 1} \mathbf{~ K}$} & \multicolumn{2}{c}{ Average } \\
\cline { 2 - 5 } & $\mathbf{M D}$ & $\mathbf{M D}+\mathbf{Y H}$ & $\mathbf{M D}$ & $\mathbf{M D}+\mathbf{Y H}$ & $\mathbf{M D}$ & $\mathbf{M D}+\mathbf{Y H}$ \\
\hline GROMOS & 1.88 & 0.85 & 1.67 & 1.33 & 1.78 & 1.09 \\
NERD & 1.67 & 0.91 & 1.56 & 1.32 & 1.62 & 1.11 \\
OPLS AA & 2.24 & 1.18 & 2.07 & 1.75 & 2.16 & 1.46 \\
OPLS UA & 2.29 & 1.26 & 2.32 & 1.56 & 2.31 & 1.41 \\
\hline
\end{tabular}

Table 2: Comparison of the AAD on the isotherms for the self-diffusivity of n-hexane(2) without (MD) and with the Yeh-Hummer correction (MD+YH), for different force fields.

$\mathrm{AAD}$ is calculated over four compositions and three pressures.

\begin{tabular}{lcc|cc|cc}
\cline { 2 - 7 } & \multicolumn{5}{c}{$D_{2} A A D / 10^{-9} m^{2} s^{-1}$} \\
\cline { 2 - 8 } & $\mathbf{T}=\mathbf{3 0 3 . 2} \mathbf{~ K}$ & \multicolumn{2}{c}{$\mathbf{T}=\mathbf{3 3 3 . 1} \mathbf{~ K}$} & \multicolumn{2}{c}{ Average } \\
\cline { 2 - 5 } & $\mathbf{M D}$ & $\mathbf{M D}+\mathbf{Y H}$ & $\mathbf{M D}$ & $\mathbf{M D}+\mathbf{Y H}$ & MD & MD+YH \\
\hline GROMOS & 1.03 & 0.50 & 0.81 & 1.16 & 0.92 & 0.83 \\
NERD & 0.66 & 1.27 & 0.85 & 2.03 & 0.76 & 1.65 \\
OPLS AA & 1.84 & 0.75 & 1.64 & 1.34 & 1.74 & 1.04 \\
OPLS UA & 1.11 & 0.43 & 0.96 & 1.22 & 1.04 & 0.83 \\
\hline
\end{tabular}

Figures 5 and 6 summarize all the calculated self-diffusion coefficients and compare them with experimental data, showing that the coefficients calculated without the Yeh-Hummer correction, and thus subject to finite-size effects, are not always underestimated. It is possible to notice in Figure 6(b) that, for high n-hexane diffusivities (high temperatures), all force fields provide highly overestimated coefficients after correction, since many values without correction are 
already above the experimental data. However, it is important to emphasize that the results without correction depend on the size of the simulation box, thus exhibiting an undesirable lack of consistency. The actual results provided by a force field should be as close as possible to the thermodynamic limit, which is only achieved by considering the Yeh-Hummer correction factor. Therefore, even when the Yeh-Hummer factor seems to make the results depart from the experimental data, its use is essential to correct the coefficients and make them consistent, since the force fields themselves are not capable of completely eliminate the influence of the finite-size effect on self-diffusion. In the event that the corrected coefficients are greatly overestimated when compared to experimental data, it is important to clarify that the poor quality of the results is not a fault of the Yeh-Hummer correction, but of the force field itself.
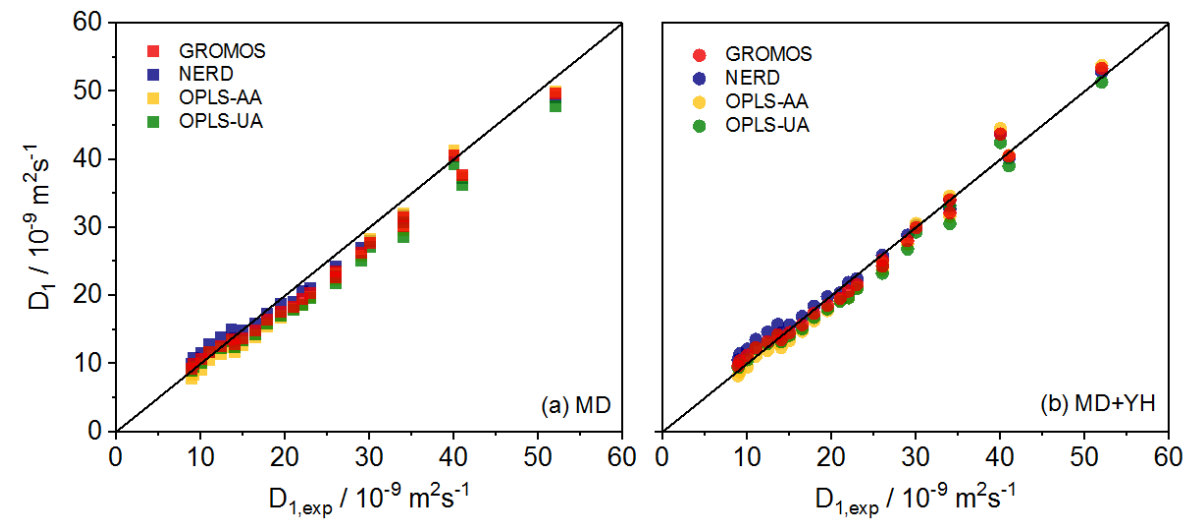

Figure 5: Self-diffusion coefficients of methane(1) provided by different force fields, over two temperatures, three pressures and four compositions. (a) $\square$ Molecular Dynamics; (b) $\bigcirc$ Molecular Dynamics with the Yeh-Hummer correction; Solid line (-): perfect agreement with experimental data. 

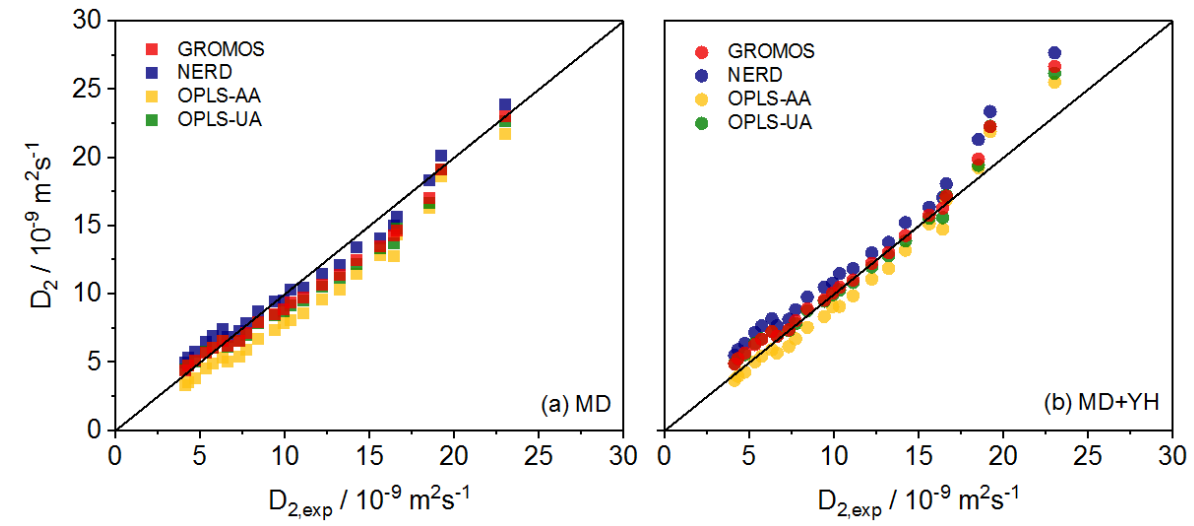

Figure 6: Self-diffusion coefficients of n-hexane(2) provided by different force field, over two temperatures, three pressures and four compositions. (a) $\square$ Molecular Dynamics; (b) $\bigcirc$ Molecular Dynamics with the Yeh-Hummer correction; Solid line (-): perfect agreement with experimental data.

On average, the GROMOS force field along with the Yeh-Hummer factor seemed to be the most accurate choice for the calculation of the self-diffusion coefficients of methane/n-hexane mixtures under the evaluated conditions, since it better approached the experimental data and presented AAD's among the lowest ones for both methane and n-hexane. It should be noted, however, that no force field provided spurious or very wrong self-diffusion coefficients under the evaluated high pressure conditions.

\subsection{Self-diffusion and the effect of force fields}

We carried out complementary analysis in order to better understand the differences in self-diffusion coefficient results provided by different force fields and the reasons why they occur. We noticed that, in most cases, the united-atom force fields (namely GROMOS, NERD, and OPLS-UA) provided higher selfdiffusion coefficients than the values obtained with the only tested all-atom force field (OPLS-AA). Figures 7 and 8 show the Average Deviation (AD) of the selfdiffusion coefficients over all evaluated temperatures and pressures as a function of composition, for all tested force fields. The $\mathrm{AD}$ values were calculated for results with the same deviation behavior, in order to avoid error canceling by 
adding positive and negative values. It is possible to notice that the all-atom force field, on average, underestimates the self-diffusion further than the unitedatom force fields. This result is expected because all-atom models tend to have less mobility due to the existence of more modes of movement besides translation (more degrees of freedom), whereas the coarse-grained models tend to have higher self-diffusive mobility.

Figure 7 shows, however, that at high methane concentrations an opposite behavior is observed for methane self-diffusivity, that is, the all-atom force field provided larger self-diffusion coefficients than the united atoms models. This unexpected result is likely related to the so-called caging effect, which can occur under certain conditions of high pressures [36. In general, the caging effect occurs at high densities because molecules in coarse-grained force fields tend to become more "packed" or "caged" by the molecules around them, whereas all-atom molecules gain a greater degree of mobility due to asymmetries and the existence of empty volumes, caused by their non-spherical forms [36, 57]. In our case, it is likely that this counter-intuitive effect occurs due to the large amount of methane in the system, which is treated as a single sphere in the united atoms force fields and, therefore, enables a denser packing than it does in the all-atom model, especially at temperatures which are not too high. Figure 9. which contains radial distribution functions for methane(1)-methane(1) pairs obtained with all models, shows that OPLS-AA seems to generate the pack with the least local density, even for the lowest evaluated methane concentration $\left(x_{1}\right.$ $=0.27$. By local density we mean the amount of molecules surrounding a certain central molecule, which creates a local fluid structure that impairs the self-diffusion. Thus, when the system becomes richer in methane $\left(x_{1}=0.88\right)$, the sparser local packing provided by OPLS-AA is sufficient to make the methane molecules diffuse more rapidly than in the coarse-grained model systems. 


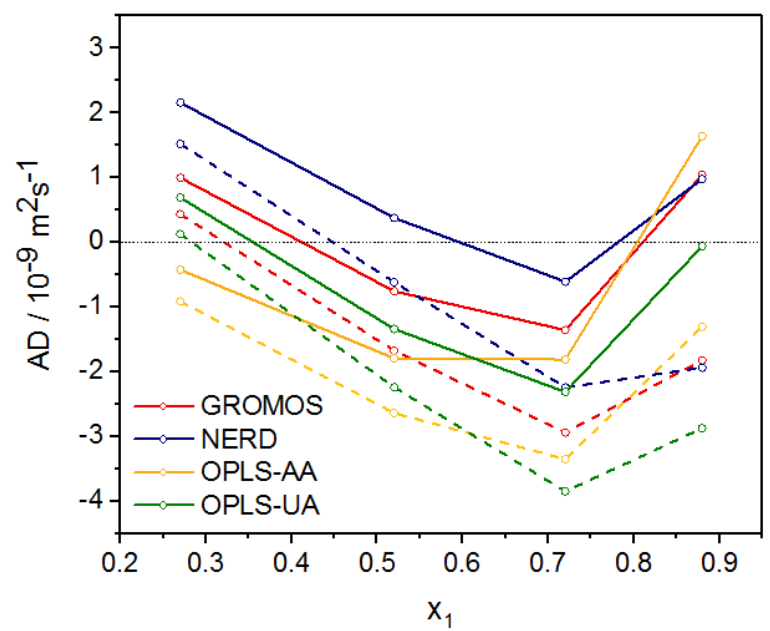

Figure 7: Comparison of the Average Deviation (AD) of self-diffusivity of methane(1) for different force fields. AD is calculated over three pressures and two temperatures. Dashed lines (--): raw molecular dynamics results; Solid lines (-): molecular dynamics results with the Yeh-Hummer correction.

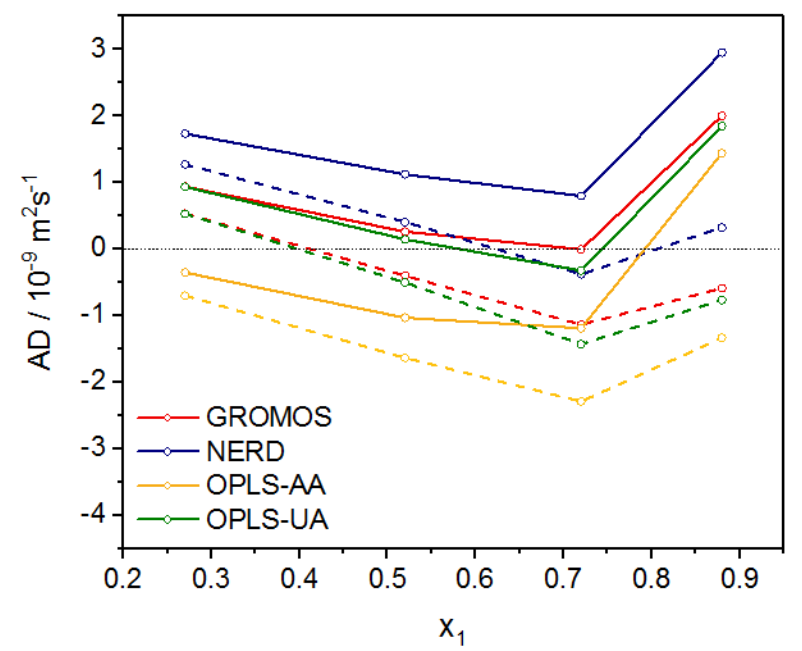

Figure 8: Comparison of the Average Deviation (AD) of self-diffusivity of n-hexane(2) for different force fields. AD is calculated over three pressures and two temperatures. Dashed lines (--): raw molecular dynamics results; Solid lines $(-)$ : molecular dynamics results with the Yeh-Hummer correction. 
As seen thus far, two important aspects that influence the self-diffusivity are the local structure of the fluid and the conformation of the molecules. Figures 9 to 11 show radial distribution functions (RDF) based on the center-of-mass positions of the molecules, aiming at highlighting the differences in the local structure of these mixtures, with $x_{1}=0.27$ at $40 \mathrm{MPa}$ and $303.2 \mathrm{~K}$, resulting from various force fields. Despite the few differences observed in Figure 9 on the local methane-methane structure, Figures 10 and 11 show that different force fields provide very distinct fluid structures when considering n-hexane, in both $g_{12}(r)$ and $g_{22}(r)$. We found out that the main reason for this disparity lies in the representation of the $\mathrm{n}$-hexane dihedral potential in each force field, which has direct implications in the flexibility of the n-alkane chain. In the literature, it has been reported that the flexibility of a molecule affects its self-diffusivity, since molecule which are more flexible usually tend to undergo smaller drag forces and exhibit faster diffusion [58, 59]. Also, according to Feng et al. (2012), 265 under certain conditions the n-alkanes chains may exist in a coiled state, which can slow down the self-diffusion process and cause certain force fields, such as OPLS-UA, to overestimate the self-diffusivity by facing difficulty in reproducing the appropriate coiled-chain conformation. 


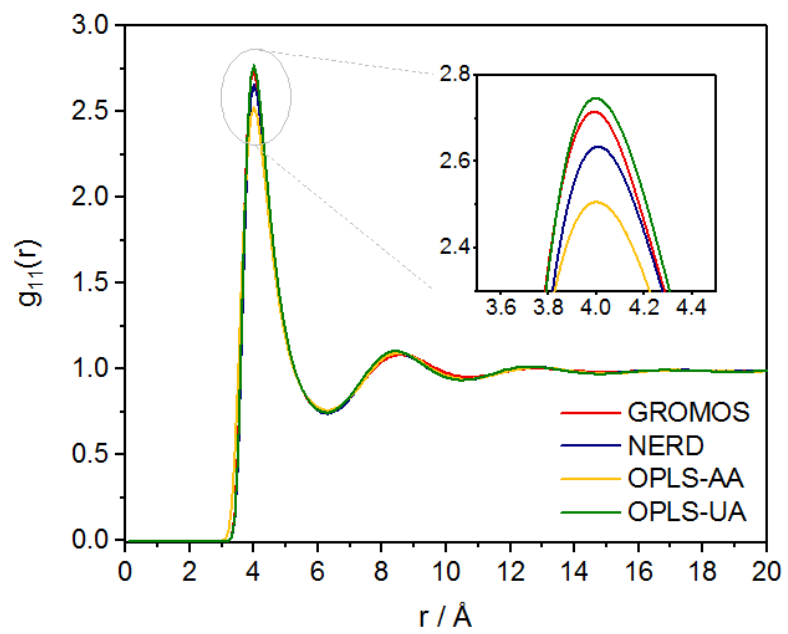

Figure 9: Radial Distribution Function $g_{11}(\mathrm{r})$ of methane-methane obtained with different force fields, for mixture with $x_{1}=0.27, \mathrm{P}=40 \mathrm{MPa}$ and $\mathrm{T}=303.2 \mathrm{~K}$.

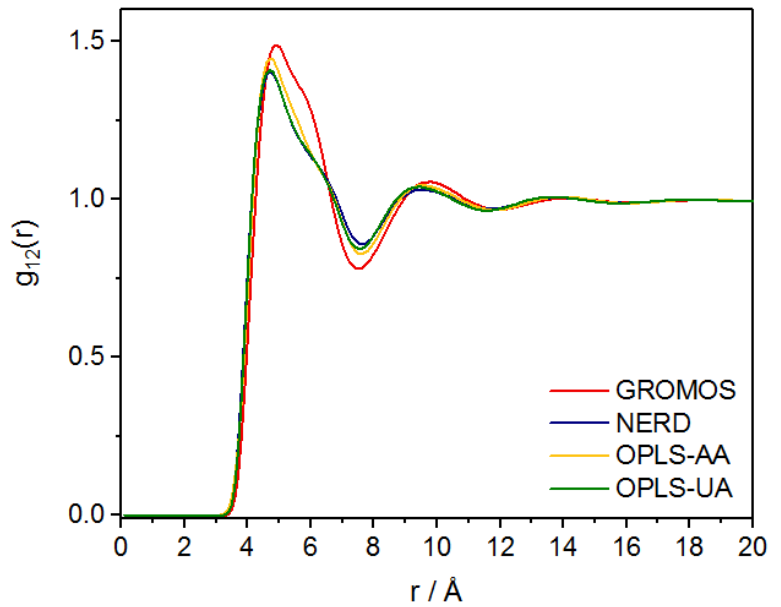

Figure 10: Radial Distribution Function $g_{12}(\mathrm{r})$ of methane-n-hexane obtained with different force fields, for mixture with $x_{1}=0.27, \mathrm{P}=40 \mathrm{MPa}$ and $\mathrm{T}=303.2 \mathrm{~K}$. 


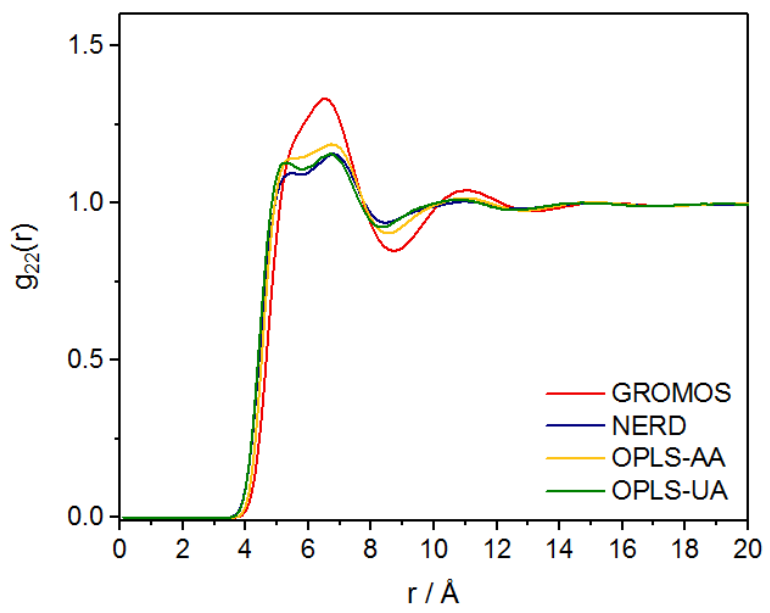

Figure 11: Radial Distribution Function $g_{22}(\mathrm{r})$ of n-hexane-n-hexane obtained with different force fields, for mixture with $x_{1}=0.27, \mathrm{P}=40 \mathrm{MPa}$ and $\mathrm{T}=303.2 \mathrm{~K}$.

Figure 12 shows the end-to-end distance distribution of n-hexane in the mix-

ture with $x_{1}=0.27$, at $40 \mathrm{MPa}$ and $303.2 \mathrm{~K}$. We did not observe significant differences in these profiles with variation of the system composition. The GROMOS force field presented the most flexible and also the most compact n-hexane structure, thus justifying the high local densities observed in Figures 10 and 11 . That is, the smaller end-to-end distance of the GROMOS n-hexane molecules gives rise to a denser packing. Both NERD and OPLS-UA presented a trimodal distribution that reveals more rigid aliphatic chains than those obtained with GROMOS. At the opposite end, the OPLS-AA force field seems to present the most rigid n-hexane structure and the least proportional trimodal distribution, with a very large peak near the distance that corresponds to a fully stretched aliphatic chain. This result of OPLS-AA is quite different from the others and does not seem to be the most appropriate distribution for n-hexane 60, principally under such temperature and pressure conditions.

In the literature, GROMOS is reported as a force field that adequately represents the dihedral distribution of alkanes, having already undergone corrections to improve the quality of the representations [42] and to correctly pre- 


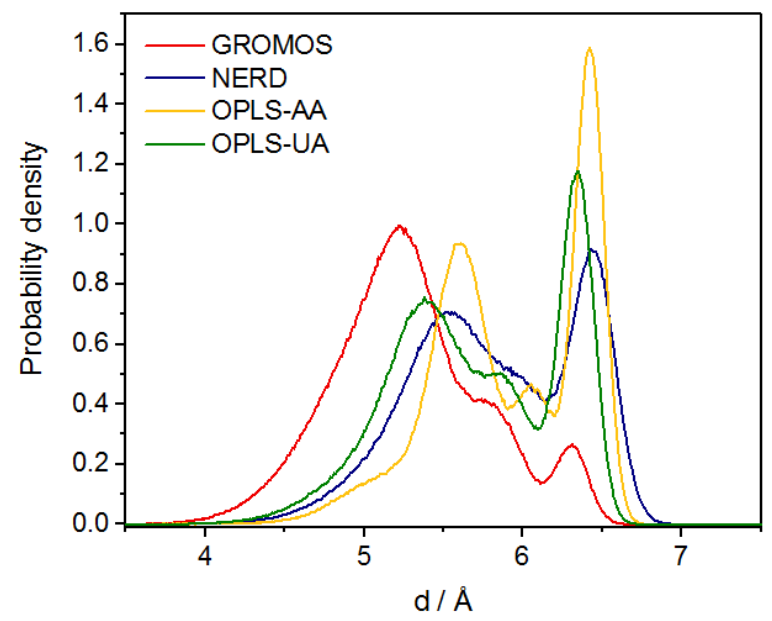

Figure 12: N-hexane end-to-end distance distribution obtained with different force fields, for mixture with $x_{1}=0.27, \mathrm{P}=40 \mathrm{MPa}$ and $\mathrm{T}=303.1 \mathrm{~K}$.

dict gauche/trans ratios. This probably contributes to the finding that the GROMOS force field, when associated with the Yeh-Hummer correction factor, presents good self-diffusivity results in comparison to the experimental data. Conversely, there are reports in the literature showing that the OPLS-AA force field does not adequately represent the alkane dihedral distributions, leading to the proposal of some corrected force fields such as L-OPLS-AA 61 and an improved OPLS-AA for peptides and proteins 62. However, even with a less consistent dihedral distribution, the OPLS-AA force field did not produce poor self-diffusion results. In this case, it is likely that the parameterization of OPLS-AA compensates errors and provides results of self-diffusion coefficients with reasonable accuracy despite its poorer representation of molecular conformations.

\section{Conclusions}

We performed several simulations of methane/n-hexane mixtures at high pressures in order to calculate thermodynamic and transport properties by means of molecular dynamics, with emphasis on self-diffusion. In general, the 
results we obtained showed good agreement with experimental data. It has been found that under reservoir conditions the system composition, temperature, and pressure exert a great influence on self-diffusion because most of the simulated systems are relatively close to their critical regions or are already in the supercritical state. The GROMOS force field with the Yeh-Hummer correction proved to be the most accurate force field for the computation of self-diffusion coefficients for the studied system at high pressures.

The Yeh-Hummer correction factor, whose purpose is to mitigate the finitesize effects of the simulation boxes in the calculation of the self-diffusion coefficient, presents an excellent consistency in the determination of the self-diffusion coefficient independently of the system size, leading the results closer to the thermodynamic limit. However, eventually the employed force field may provide an already overestimated value of self-diffusivity, and thus the addition of the Yeh-Hummer factor may worsen the results with respect to the experimental data. This can happen for several reasons, such as the fact that the force field has not been parameterized considering the finite-size effect or the fact that the model does not adequately represent the conformations of the n-alkanes. However, even in these cases, the use of the correction factor is recommended. Unless the simulation is carried out in a very large box, only the corrected self-diffusion coefficient is sufficiently size-independent to be considered as the actual result of a force field. Our results suggest that an effort should be made to parameterize force fields by taking this correction into account.

We also found out that the choice of a united-atom force field can lead to a sort of caging effect under some circumstances, causing a tendency of producing a slow self-diffusion process in comparison to all-atom models. In addition, the choice of the force field may also affect self-diffusion due to its ability, or lack thereof, of reproducing the dihedral distribution of a molecule. In this sense, we noticed that the OPLS-AA force field presents an end-to-end profile for n-hexane which is far from the expected one, and quite different from the outcome of the other tested force fields. Nevertheless, its self-diffusion coefficient results are not awfully compromised, perhaps due to some compensation of errors imprinted in 
its whole set of parameters.

\section{Acknowledgements}

We are grateful for the financial support provided by CAPES (finance code 001), by Petrobras (project code CENPES 20934-6), by CNPq and by FAPERJ. We are also grateful for the computational resources provided by the Lobo Carneiro Supercomputer Facility at the Federal University of Rio de Janeiro, Brazil.

\section{References}

[1] L. Wei-Zhong, C. Cong, Y. Jian, Molecular Dynamics Simulation of SelfDiffusion Coefficient and Its Relation with Temperature Using Simple Lennard-Jones Potential, Heat Transfer-Asian Research 37 (2) (2008) 8693. doi:10.1002/htj.20191.

[2] A. L. Hines, R. N. Maddox, Mass Transfer: Fundamentals and applications, first edit Edition, Prentice Hall, New Jesery, 1984.

[3] R. B. Bird, W. E. Stewart, E. N. Lightfoot, Transport phenomena, second edi Edition, John Wiley \& Sons, INC., New York, 2002.

[4] T. L. Bergman, A. S. Lavine, F. P. Incropera, D. P. Dewitt, Fundamentals of Heat and Mass Transfer, seventh ed Edition, John Wiley \& Sons, INC., 2014.

[5] R. O. Espósito, P. H. R. Alijó, J. A. Scilipoti, F. W. Tavares, Compositional Grading in Oil and Gas Reservoirs, Elsevier, 2017. doi:10.1016/ b978-0-12-812452-9.00009-5.

[6] S. Srinivasan, M. Z. Saghir, Experimental approaches to study thermodiffusion - A review, International Journal of Thermal Sciences 50 (7) (2011) 1125-1137. doi:10.1016/j.ijthermalsci.2011.02.022 
[7] O. A. Moultos, Y. Zhang, I. N. Tsimpanogiannis, I. G. Economou, E. J. Maginn, System-size corrections for self-diffusion coefficients calculated from molecular dynamics simulations: The case of $\mathrm{CO}_{2}$, n-alkanes, and poly(ethylene glycol) dimethyl ethers, Journal of Chemical Physics 145 (7). doi:10.1063/1.4960776.

[8] F. Cecconi, M. Cencini, M. Falcioni, A. Vulpiani, Brownian motion and diffusion: From stochastic processes to chaos and beyond, Chaos 15 (2). arXiv:0407029, doi:10.1063/1.1832773.

[9] J. Piasecki, Centenary of Marian Smoluchowski's Theory of Brownian Motion, Acta Physica Polonica B 38 (5) (2007) 1623-1629.

[10] P. Hänggi, F. Marchesoni, Introduction: 100 years of Brownian motion, Chaos 15 (2). arXiv:0502053, doi:10.1063/1.1895505.

370 [11] T. Gross, J. Buchhauser, H. D. Lüdemann, Self-diffusion in fluid carbon dioxide at high pressures, Journal of Chemical Physics 109 (11) (1998) 4518-4522. doi:10.1063/1.477055

[12] T. L. James, G. G. McDonald, Measurement of the self-diffusion coefficient of each component in a complex system using pulsed-gradient fourier transform NMR, Journal of Magnetic Resonance (1969) 11 (1) (1973) 5861. doi:10.1016/0022-2364(73)90081-4.

[13] M. Helbaek, B. Hafskjold, D. K. Dysthe, G. H. Sørland, Self-Diffusion Coefficients of Methane or Ethane Mixtures with Hydrocarbons at High Pressure by NMR, Journal of Chemical \& Engineering Data 41 (3) (1996) 598-603. doi:10.1021/je950293p.

[14] H. Higashi, Y. Iwai, Y. Arai, Calculation of Self-Diffusion and Tracer Diffusion Coefficients near the Critical Point of Carbon Dioxide Using Molecular Dynamics Simulation, Industrial \& Engineering Chemistry Research 39 (12) (2000) 4567-4570. doi:10.1021/ie000173x. 
[23] E. J. Maginn, R. A. Messerly, D. J. Carlson, D. R. Roe, J. R. Elliott, Best Practices for Computing Transport Properties 1. Self-Diffusivity

[15] H. Feng, W. Gao, J. Nie, J. Wang, X. Chen, L. Chen, X. Liu, H. D. Lüdemann, Z. Sun, MD simulation of self-diffusion and structure in some nalkanes over a wide temperature range at high pressures, Journal of Molecular Modeling 19 (1) (2013) 73-82. doi:10.1007/s00894-012-1514-0.

[16] A. Einstein, Über die von der molekularkinetischen Theorie der Wärme geforderte Bewegung von in Flüssigkeiten suspendierten Teilchen, Ann. d. Phys. 322 (8) (1905) 549-560. doi:10.1002/andp.19053220806.

[17] D. Frenkel, B. Smit, Understanding Molecular Simulation, Academic Press, New York, 2002.

[18] R. Kubo, Statistical-Mechanical Theory of Irreversible Processes., Journal of the Physical Society of Japan 12 (6) (1957) 570-586. doi:10.1143/ JPSJ.12.570,

[19] M. S. Green, Markoff random processes and the statistical mechanics of time-dependent phenomena. II. Irreversible processes in fluids, The Journal of Chemical Physics 22 (3) (1954) 398-413. doi:10.1063/1.1740082.

[20] Y. Zhou, G. H. Miller, Green-Kubo formulas for mutual diffusion coefficients in multicomponent systems, Journal of Physical Chemistry 100 (13) (1996) 5516-5524. arXiv:0604102, doi:10.1021/jp9533739.

[21] J. Hansen, I. McDonald, Theory of simple liquids, 3rd Edition, Academic Press, 2005.

[22] H. Ohta, S. Hamaguchi, Molecular dynamics evaluation of self-diffusion in Yukawa systems, Physics of Plasmas 7 (11) (2000) 4506-4514. doi: $10.1063 / 1.1316084$ and Viscosity from Equilibrium Molecular Dynamics [Article v1.0], Living Journal of Computational Molecular Science 1 (1) (2018) 1-20. doi: 10.33011/livecoms.1.1.6324 
[24] D. Keffer, The Working Man's Guide to Obtaining Self Diffusion Coefficients from Molecular Dynamics Simulations, http://www.cs.unc.edu/ Research/nbody/pubs/external/Keffer/selfD.pdf (2002).

[25] D. Dubbeldam, D. C. Ford, D. E. Ellis, R. Q. Snurr, A new perspective on the order-n algorithm for computing correlation functions, Molecular Simulation 35 (12-13) (2009) 1084-1097. doi:10.1080/08927020902818039.

[26] B. Dünweg, K. Kremer, Microscopic Verification of Dynamic Scaling in Dilute Polymer-Solutions - a Molecular-Dynamics Simulation, Phys. Rev. Lett. 66 (3) (1991) 2996-2999.

[27] J. G. Kirkwood, J. Riseman, The intrinsic viscosities and diffusion constants of flexible macromolecules in solution, The Journal of Chemical Physics 16 (6) (1948) 565-573. doi:10.1063/1.1746947.

${ }_{425}$ [28] B. Dünweg, K. Kremer, Molecular dynamics simulation of a polymer chain in solution, The Journal of Chemical Physics 99 (9) (1993) 6983-6997. doi:10.1063/1.465445.

[29] I. C. Yeh, G. Hummer, System-size dependence of diffusion coefficients and viscosities from molecular dynamics simulations with periodic boundary conditions, Journal of Physical Chemistry B 108 (40) (2004) 15873-

a 15879. arXiv:10.1021/nl3012853/NanoLett.2012,12,36023608, doi: $10.1021 / \mathrm{jp} 0477147$

[30] H. Hasimoto, On the periodic fundamental solutions of the Stokes equations and their application to viscous, J. Fluid Mech. 5 (1958) (1958) 317-328.

${ }_{435}$ [31] G. Hummer, L. R. Pratt, A. E. García, Hydration free energy of wa口 ter, Journal of Physical Chemistry 99 (38) (1995) 14188-14194. arXiv: 9507004, doi:10.1021/j100038a062

[32] R. E. Zeebe, On the molecular diffusion coefficients of dissolved $\mathrm{CO}_{2}$, $\mathrm{HCO}_{3}^{-}$, and $\mathrm{CO}_{3}^{2-}$ and their dependence on isotopic mass, Geochimica et 
Cosmochimica Acta 75 (9) (2011) 2483-2498. doi:10.1016/j.gca.2011. 02.010

[33] R. O. Sokolovskii, M. Thachuk, G. N. Patey, Tracer diffusion in hard sphere fluids from molecular to hydrodynamic regimes, Journal of Chemical Physics 125 (20). doi:10.1063/1.2397074.

[34] A. Botan, V. Marry, B. Rotenberg, Diffusion in bulk liquids: Finite-size effects in anisotropic systems, Molecular Physics 113 (17-18) (2015) 26742679. doi:10.1080/00268976.2015.1021730.

[35] D. M. Heyes, M. J. Cass, J. G. Powles, W. A. Evans, Self-diffusion coefficient of the hard-sphere fluid: System size dependence and empirical correlations, Journal of Physical Chemistry B 111 (6) (2007) 1455-1464. doi:10.1021/jp067373s.

[36] C. G. Aimoli, E. J. Maginn, C. R. A. Abreu, Transport properties of carbon dioxide and methane from molecular dynamics simulations, The Journal of Chemical Physics 141 (13) (2014) 134101. doi:10.1063/1.4896538.

[37] Y. M. Gonçalves, C. Senac, P. F. Fuchs, P. H. Hünenberger, B. A. Horta, Influence of the Treatment of Nonbonded Interactions on the Thermodynamic and Transport Properties of Pure Liquids Calculated Using the 2016H66 Force Field, Journal of Chemical Theory and Computation 15 (3) (2019) 1806-1826. doi:10.1021/acs.jctc.8b00425.

[38] Z. Zhang, Z. Duan, An optimized molecular potential for carbon dioxide, Journal of Chemical Physics 122 (21). doi:10.1063/1.1924700

[39] P. Ungerer, C. Beauvais, J. Delhommelle, A. Boutin, B. Rousseau, A. H. Fuchs, Optimization of the anisotropic united atoms intermolecular potential for n-alkanes, Journal of Chemical Physics 112 (12) (2000) 5499-5510. doi:10.1063/1.481116. 
[40] W. L. Jorgensen, J. D. Madura, C. J. Swenson, Optimized Intermolecular Potential Functions for Liquid Hydrocarbons, Journal of the American Chemical Society 106 (22) (1984) 6638-6646. doi:10.1021/ja00334a030

[41] W. L. Jorgensen, D. S. Maxwell, J. Tirado-Rives, Development and Testing of the OPLS All-Atoms Force Field on Conformational Energetics and Properties of Organic Liquids, J. Am. Chem. Soc. 118 (1) (1990) 1-12. doi:10.1021/ja9621760.

[42] L. D. Schuler, W. F. Van Gunsteren, On the choice of dihedral angle potential energy functions for n-alkanes, Molecular Simulation 25 (5) (2000) 301-319. doi:10.1080/08927020008024504.

[43] L. D. Schuler, X. Daura, W. F. Van Gunsteren, An improved GROMOS96 force field for aliphatic hydrocarbons in the condensed phase, Journal of

■ Computational Chemistry 22 (11) (2001) 1205-1218. doi:10.1002/jcc. 1078.

[44] C. Oostenbrink, A. Villa, A. E. Mark, W. F. Van Gunsteren, A biomolecular force field based on the free enthalpy of hydration and solvation: The GROMOS force-field parameter sets 53A5 and 53A6, Journal of Computational Chemistry 25 (13) (2004) 1656-1676. doi:10.1002/jcc.20090.

[45] S. M. Nath, F. A. Escobedo, J. d. Pablo, On the simulation of vapor-liquid equilibria for alkanes, Journal of Chemical Physics 108 (23) (1998) 99059911. doi:10.1063/1.476429.

[46] M. G. Martin, J. I. Siepmann, Transferable Potentials for Phase Equilibria. 1. United-Atom Description of $\mathrm{n}$-Alkanes, The Journal of Physical Chemistry B 102 (97) (1998) 2569-2577. doi:10.1021/jp972543+.

[47] B. A. Horta, P. T. Merz, P. F. Fuchs, J. Dolenc, S. Riniker, P. H. Hünenberger, A GROMOS-Compatible Force Field for Small Organic Molecules in the Condensed Phase: The 2016H66 Parameter Set, Jour- 
nal of Chemical Theory and Computation 12 (8) (2016) 3825-3850. doi: 10.1021/acs.jctc.6b00187

[48] C. R. A. Abreu, Playmol, https://github.com/atoms-ufrj/playmol (2018).

[49] S. Plimpton, Fast Parallel Algorithms for Short - Range Molecular Dynamics, Journal of Computational Physics 117 (June 1994) (1995) 1-19. arXiv:nag.2347, doi:10.1006/jcph.1995.1039.

[50] R. W. Hockney, J. W. Eastwood, Computer Simulation Using Particles, first edit Edition, New York, 1988.

[51] G. J. Martyna, M. L. Klein, M. Tuckerman, Nosé - Hoover chains : The canonical ensemble via continuous dynamics Nose-Hoover chains : The canonical ensemble via continuous dynamics, Journal of Chemical Physics 2635 (97) (1992) 2635-2643. doi:10.1063/1.463940

[52] G. J. Martyna, D. J. Tobias, M. L. Klein, Constant pressure molecular dynamics algorithms, The Journal of Chemical Physics 101 (4177) (1994) 4177-4189. arXiv:0502063v2, doi:10.1063/1.467468.

[53] M. E. Tuckerman, J. Alejandre, R. López-Rendón, A. L. Jochim, G. J. Martyna, A Liouville-operator derived measure-preserving integrator for molecular dynamics simulations in the isothermal-isobaric ensemble, Journal of Physics A: Mathematical and General 39 (5629) (2006) 5629-5651. doi:10.1088/0305-4470/39/19/S18.

[54] O. Kunz, W. Wagner, The GERG-2008 wide-range equation of state for natural gases and other mixtures: An expansion of GERG-2004, Journal of

口. Chemical and Engineering Data 57 (11) (2012) 3032-3091. doi:10.1021/ je300655b.

[55] E. Lemmon, M. Huber, M. McLinden, Reference Fluid Thermodynamic and Transport Properties -REFPROP, Version 7.0. - User's Guide. 
[56] J. E. Basconi, M. R. Shirts, Effects of temperature control algorithms on transport properties and kinetics in molecular dynamics simulations, Journal of Chemical Theory and Computation 9 (7) (2013) 2887-2899. doi:10.1021/ct400109a.

[57] S. Munejiri, T. Masaki, T. Itami, F. Shimojo, K. Hoshino, Static and dynamic structure and the atomic dynamics of liquid Ge from first-principles molecular-dynamics simulations, Physical Review B - Condensed Matter and Materials Physics 77 (14206) (2008) 1-12. doi:10.1103/PhysRevB. 77.014206 .

[58] J. Wang, H. Zhong, H. Feng, W. Qiu, L. Chen, Molecular dynamics simulation of diffusion coefficients and structural properties of some alkylbenzenes in supercritical carbon dioxide at infinite dilution, Journal of Chemical Physics 140 (10). doi:10.1063/1.4867274.

[59] F. G. Pazzona, B. J. Borah, P. Demontis, G. B. Suffritti, S. Yashonath, A comparative molecular dynamics study of diffusion of n-decane and 3methyl pentane in y zeolite, Journal of Chemical Sciences 121 (5) (2009) 921-927. doi:10.1007/s12039-009-0109-5

[60] Y. Huang, H. Wang, An investigation of the conformational behavior of nhexane adsorbed in zeolites by FT-Raman spectroscopy, Langmuir 19 (23) (2003) 9706-9713. doi:10.1021/la030115r.

[61] M. J. Robertson, J. Tirado-Rives, W. L. Jorgensen, Improved Peptide and Protein Torsional Energetics with the OPLS-AA Force Field, Journal of Chemical Theory and Computation 11 (7) (2015) 3499-3509. doi:10. 1021/acs.jctc.5b00356.

[62] S. W. I. Siu, K. Pluhackova, R. A. Böckmann, Optimization of the OPLSAA Force Field for Long Hydrocarbons, Journal of Chemical Theory and Computation 8 (2012) 1459-1470. doi:10.1021/ct200908r. 


\section{Supplementary Material}

Table S1: Density and viscosity results obtained with GROMOS Force Field for methane(1)/n-hexane(2) mixtures

\begin{tabular}{|c|c|c|c|c|c|c|c|c|}
\hline \multirow{2}{*}{$\mathbf{T} / \mathbf{K}$} & \multirow{2}{*}{$x_{1}$} & \multirow{2}{*}{$\mathrm{P} / \mathrm{MPa}$} & \multicolumn{3}{|c|}{$\rho / k \cdot m^{-3}$} & \multicolumn{3}{|c|}{$\eta / 10^{-4}$ Pa.s } \\
\hline & & & MD & GERG-2008 & Dev. (\%) & MD & REFPROP & Dev. (\%) \\
\hline \multirow{12}{*}{303.2} & 0.27 & 30 & $638.7 \pm 3.8$ & 639.5 & $-0.13 \%$ & $2.065 \pm 0.078$ & 2.479 & $-16.69 \%$ \\
\hline & 0.27 & 40 & $647.7 \pm 3.6$ & 648.9 & $-0.18 \%$ & $2.304 \pm 0.120$ & 2.685 & $-14.16 \%$ \\
\hline & 0.27 & 50 & $658.1 \pm 3.4$ & 657.3 & $0.13 \%$ & $2.448 \pm 0.046$ & 2.887 & $-15.19 \%$ \\
\hline & 0.52 & 30 & $568.7 \pm 4.5$ & 564.8 & $0.69 \%$ & $1.383 \pm 0.092$ & 1.461 & $-5.35 \%$ \\
\hline & 0.52 & 40 & $580.5 \pm 4.3$ & 577.5 & $0.53 \%$ & $1.484 \pm 0.009$ & 1.597 & $-7.08 \%$ \\
\hline & 0.52 & 50 & $590.4 \pm 4.2$ & 588.4 & $0.34 \%$ & $1.647 \pm 0.028$ & 1.725 & $-4.54 \%$ \\
\hline & 0.72 & 30 & $467.5 \pm 5.9$ & 463.2 & $0.92 \%$ & $0.810 \pm 0.051$ & 0.806 & $0.51 \%$ \\
\hline & 0.72 & 40 & $486.6 \pm 5.3$ & 481.6 & $1.04 \%$ & $0.980 \pm 0.041$ & 0.901 & $8.75 \%$ \\
\hline & 0.72 & 50 & $501.7 \pm 4.8$ & 496.3 & $1.09 \%$ & $1.059 \pm 0.008$ & 0.986 & $7.35 \%$ \\
\hline & 0.88 & 30 & $336.9 \pm 6.5$ & 335.6 & $0.39 \%$ & $0.446 \pm 0.019$ & 0.431 & $3.47 \%$ \\
\hline & 0.88 & 40 & $367.8 \pm 6.0$ & 364.2 & $1.01 \%$ & $0.525 \pm 0.021$ & 0.501 & $4.79 \%$ \\
\hline & 0.88 & 50 & $388.6 \pm 5.4$ & 384.9 & $0.98 \%$ & $0.592 \pm 0.012$ & 0.561 & $5.42 \%$ \\
\hline \multirow{12}{*}{333.1} & 0.27 & 30 & $619.1 \pm 4.1$ & 615.1 & $0.64 \%$ & $1.746 \pm 0.100$ & 1.985 & $-12.04 \%$ \\
\hline & 0.27 & 40 & $628.7 \pm 3.9$ & 626.1 & $0.42 \%$ & $2.028 \pm 0.071$ & 2.161 & $-6.15 \%$ \\
\hline & 0.27 & 50 & $638.4 \pm 3.7$ & 635.8 & $0.40 \%$ & $2.165 \pm 0.026$ & 2.330 & $-7.11 \%$ \\
\hline & 0.52 & 30 & $541.1 \pm 5.0$ & 536.7 & $0.82 \%$ & $1.147 \pm 0.030$ & 1.193 & $-3.93 \%$ \\
\hline & 0.52 & 40 & $555.7 \pm 4.8$ & 552.0 & $0.68 \%$ & $1.300 \pm 0.026$ & 1.318 & $-1.37 \%$ \\
\hline & 0.52 & 50 & $433.9 \pm 6.4$ & 564.8 & $0.54 \%$ & $1.380 \pm 0.018$ & 1.433 & $-3.68 \%$ \\
\hline & 0.72 & 30 & $457.7 \pm 5.7$ & 430.0 & $0.91 \%$ & $0.664 \pm 0.006$ & 0.665 & $-0.07 \%$ \\
\hline & 0.72 & 40 & $475.6 \pm 5.4$ & 452.9 & $1.06 \%$ & $0.775 \pm 0.017$ & 0.753 & $2.97 \%$ \\
\hline & 0.72 & 50 & $336.3 \pm 6.2$ & 470.6 & $1.08 \%$ & $0.847 \pm 0.016$ & 0.831 & $1.89 \%$ \\
\hline & 0.88 & 30 & $361.6 \pm 5.6$ & 299.2 & $-0.41 \%$ & $0.365 \pm 0.012$ & 0.369 & $-0.89 \%$ \\
\hline & 0.88 & 40 & $336.3 \pm 6.2$ & 333.8 & $0.76 \%$ & $0.449 \pm 0.006$ & 0.435 & $3.29 \%$ \\
\hline & 0.88 & 50 & $361.6 \pm 5.6$ & 358.1 & $0.99 \%$ & $0.524 \pm 0.031$ & 0.491 & $6.79 \%$ \\
\hline
\end{tabular}


Table S2: Density and viscosity results obtained with NERD Force Field for methane(1)/n-hexane(2) mixtures

\begin{tabular}{ccc|ccc|ccc}
\hline \multirow{2}{*}{ T/K } & \multirow{2}{*}{$x_{1}$} & \multirow{2}{*}{ P/MPa } & \multicolumn{3}{|c|}{$\rho / \mathrm{kg}^{-m^{-3}}$} & \multicolumn{3}{c}{$\eta / 10^{-4}$ Pa.s } \\
\cline { 4 - 9 } & & MD & GERG-2008 & Dev. (\%) & MD & REFPROP & Dev. (\%) \\
\hline 0.27 & 30 & $619.8 \pm 3.8$ & 639.5 & $-3.09 \%$ & $1.864 \pm 0.047$ & 2.479 & $-24.80 \%$ \\
0.27 & 40 & $629.4 \pm 3.6$ & 648.9 & $-3.00 \%$ & $1.947 \pm 0.025$ & 2.685 & $-27.49 \%$ \\
0.27 & 50 & $637.9 \pm 3.5$ & 657.3 & $-2.95 \%$ & $2.145 \pm 0.068$ & 2.887 & $-25.69 \%$ \\
\hline 0.52 & 30 & $550.6 \pm 4.6$ & 564.8 & $-2.52 \%$ & $1.235 \pm 0.043$ & 1.461 & $-15.45 \%$ \\
0.52 & 40 & $563.0 \pm 4.4$ & 577.5 & $-2.50 \%$ & $1.398 \pm 0.039$ & 1.597 & $-12.46 \%$ \\
\hline 303.2 & 0.52 & 50 & $573.6 \pm 4.1$ & 588.4 & $-2.50 \%$ & $1.513 \pm 0.066$ & 1.725 & $-12.28 \%$ \\
\hline 0.72 & 30 & $456.8 \pm 5.7$ & 463.2 & $-1.39 \%$ & $0.814 \pm 0.006$ & 0.806 & $0.98 \%$ \\
0.72 & 40 & $475.3 \pm 5.2$ & 481.6 & $-1.29 \%$ & $0.890 \pm 0.026$ & 0.901 & $-1.26 \%$ \\
\hline 0.88 & 50 & $490.2 \pm 4.8$ & 496.3 & $-1.24 \%$ & $0.965 \pm 0.026$ & 0.986 & $-2.19 \%$ \\
\hline 0.88 & 40 & $334.0 \pm 6.4$ & 335.6 & $-0.46 \%$ & $0.421 \pm 0.011$ & 0.431 & $-2.22 \%$ \\
0.88 & 50 & $362.7 \pm 5.8$ & 364.2 & $-0.41 \%$ & $0.546 \pm 0.020$ & 0.501 & $8.94 \%$ \\
\hline 0.27 & $303.7 \pm 5.2$ & 384.9 & $-0.29 \%$ & $0.576 \pm 0.004$ & 0.561 & $2.72 \%$ \\
\hline 0.27 & 40 & $597.1 \pm 4.2$ & 615.1 & $-2.93 \%$ & $1.625 \pm 0.034$ & 1.985 & $-18.12 \%$ \\
0.27 & 50 & $607.9 \pm 4.0$ & 626.1 & $-2.91 \%$ & $1.717 \pm 0.057$ & 2.161 & $-20.53 \%$ \\
\hline 0.52 & 30 & $523.9 \pm 3.8$ & 635.8 & $-2.81 \%$ & $1.810 \pm 0.015$ & 2.330 & $-22.31 \%$ \\
\hline 0.52 & 40 & $538.6 \pm 4.7$ & 552.0 & $-2.43 \%$ & $1.210 \pm 0.007$ & 1.318 & $-8.19 \%$ \\
0.52 & 50 & $551.2 \pm 4.5$ & 564.8 & $-2.40 \%$ & $1.241 \pm 0.014$ & 1.433 & $-13.41 \%$ \\
\hline 0.72 & 30 & $424.1 \pm 6.2$ & 430.0 & $-1.37 \%$ & $0.654 \pm 0.018$ & 0.665 & $-1.55 \%$ \\
0.72 & 40 & $447.1 \pm 5.5$ & 452.9 & $-1.28 \%$ & $0.738 \pm 0.028$ & 0.753 & $-1.92 \%$ \\
0.72 & 50 & $465.2 \pm 5.1$ & 470.6 & $-1.13 \%$ & $0.834 \pm 0.020$ & 0.831 & $0.32 \%$ \\
\hline 0.88 & 30 & $297.8 \pm 6.6$ & 299.2 & $-0.47 \%$ & $0.356 \pm 0.016$ & 0.369 & $-3.47 \%$ \\
0.88 & 40 & $332.7 \pm 6.0$ & 333.8 & $-0.32 \%$ & $0.435 \pm 0.018$ & 0.435 & $-0.03 \%$ \\
0.88 & 50 & $357.2 \pm 5.5$ & 358.1 & $-0.26 \%$ & $0.518 \pm 0.028$ & 0.491 & $5.58 \%$ \\
\hline
\end{tabular}


Table S3: Density and viscosity results obtained with OPLS-AA Force Field for methane(1)/n-hexane(2) mixtures

\begin{tabular}{ccc|ccc|ccc}
\hline \multirow{2}{*}{ T/K } & \multirow{2}{*}{$x_{1}$} & \multirow{2}{*}{ P/MPa } & \multicolumn{3}{|c|}{$\rho / \mathrm{kg}^{-m^{-3}}$} & \multicolumn{3}{c}{$\eta / 10^{-4}$ Pa.s } \\
\cline { 4 - 9 } & & MD & GERG-2008 & Dev. (\%) & MD & REFPROP & Dev. (\%) \\
\hline 0.27 & 30 & $629.6 \pm 3.7$ & 639.5 & $-1.55 \%$ & $2.456 \pm 0.139$ & 2.479 & $-0.90 \%$ \\
0.27 & 40 & $638.2 \pm 3.6$ & 648.9 & $-1.64 \%$ & $2.677 \pm 0.019$ & 2.685 & $-0.29 \%$ \\
0.27 & 50 & $646.2 \pm 3.5$ & 657.3 & $-1.68 \%$ & $2.957 \pm 0.106$ & 2.887 & $2.44 \%$ \\
\hline 0.52 & 30 & $561.3 \pm 4.6$ & 564.8 & $-0.63 \%$ & $1.482 \pm 0.095$ & 1.461 & $1.45 \%$ \\
0.52 & 40 & $573.3 \pm 4.2$ & 577.5 & $-0.72 \%$ & $1.708 \pm 0.062$ & 1.597 & $6.93 \%$ \\
0.52 & 50 & $583.3 \pm 4.2$ & 588.4 & $-0.86 \%$ & $1.847 \pm 0.061$ & 1.725 & $7.06 \%$ \\
\hline 0.72 & 30 & $467.0 \pm 5.6$ & 463.2 & $0.81 \%$ & $0.857 \pm 0.028$ & 0.806 & $6.35 \%$ \\
0.72 & 40 & $485.6 \pm 5.1$ & 481.6 & $0.84 \%$ & $0.971 \pm 0.020$ & 0.901 & $7.77 \%$ \\
0.72 & 50 & $500.0 \pm 4.9$ & 496.3 & $0.74 \%$ & $1.064 \pm 0.099$ & 0.986 & $7.91 \%$ \\
\hline 0.88 & 30 & $342.1 \pm 6.8$ & 335.6 & $1.94 \%$ & $0.435 \pm 0.004$ & 0.431 & $0.97 \%$ \\
0.88 & 40 & $370.7 \pm 6.2$ & 364.2 & $1.80 \%$ & $0.519 \pm 0.010$ & 0.501 & $3.62 \%$ \\
0.88 & 50 & $391.8 \pm 5.4$ & 384.9 & $1.81 \%$ & $0.580 \pm 0.006$ & 0.561 & $3.26 \%$ \\
\hline 0.27 & 30 & $602.5 \pm 4.1$ & 615.1 & $-2.05 \%$ & $2.031 \pm 0.057$ & 1.985 & $2.29 \%$ \\
0.27 & 40 & $613.0 \pm 3.9$ & 626.1 & $-2.09 \%$ & $2.232 \pm 0.020$ & 2.161 & $3.30 \%$ \\
0.27 & 50 & $622.0 \pm 3.7$ & 635.8 & $-2.17 \%$ & $2.308 \pm 0.040$ & 2.330 & $-0.97 \%$ \\
\hline 0.52 & 30 & $530.3 \pm 5.2$ & 536.7 & $-1.19 \%$ & $1.253 \pm 0.039$ & 1.193 & $5.00 \%$ \\
0.52 & 40 & $544.6 \pm 4.7$ & 552.0 & $-1.33 \%$ & $1.312 \pm 0.023$ & 1.318 & $-0.45 \%$ \\
0.52 & 50 & $556.9 \pm 4.4$ & 564.8 & $-1.40 \%$ & $1.508 \pm 0.091$ & 1.433 & $5.22 \%$ \\
\hline 0.72 & 30 & $431.1 \pm 6.3$ & 430.0 & $0.26 \%$ & $0.691 \pm 0.043$ & 0.665 & $3.98 \%$ \\
0.72 & 40 & $453.9 \pm 5.7$ & 452.9 & $0.23 \%$ & $0.798 \pm 0.013$ & 0.753 & $5.99 \%$ \\
0.72 & 50 & $471.3 \pm 5.2$ & 470.6 & $0.16 \%$ & $0.868 \pm 0.027$ & 0.831 & $4.43 \%$ \\
\hline 0.88 & 30 & $303.3 \pm 6.8$ & 299.2 & $1.38 \%$ & $0.358 \pm 0.022$ & 0.369 & $-2.88 \%$ \\
0.88 & 40 & $338.2 \pm 5.9$ & 333.8 & $1.34 \%$ & $0.433 \pm 0.007$ & 0.435 & $-0.42 \%$ \\
0.88 & 50 & $362.7 \pm 5.6$ & 358.1 & $1.28 \%$ & $0.509 \pm 0.020$ & 0.491 & $3.64 \%$ \\
\hline
\end{tabular}


Table S4: Density and viscosity results obtained with OPLS-UA Force Field for methane(1)/n-hexane(2) mixtures

\begin{tabular}{ccc|ccc|ccc}
\hline \multirow{2}{*}{ T/K } & \multirow{2}{*}{$x_{1}$} & \multirow{2}{*}{$\mathbf{P} / \mathbf{M P a}$} & \multicolumn{3}{|c|}{$\rho /{\mathrm{kg} . \mathrm{m}^{-3}}$} & \multicolumn{3}{c}{$\eta / 10^{-4}$ Pa.s } \\
\cline { 4 - 9 } & & MD & GERG-2008 & Dev. (\%) & MD & REFPROP & Dev. (\%) \\
\hline 0.27 & 30 & $645.3 \pm 3.6$ & 639.5 & $0.90 \%$ & $2.128 \pm 0.069$ & 2.479 & $20.67 \%$ \\
0.27 & 40 & $653.5 \pm 3.5$ & 648.9 & $0.70 \%$ & $2.269 \pm 0.152$ & 2.685 & $19.39 \%$ \\
0.27 & 50 & $660.6 \pm 3.4$ & 657.3 & $0.51 \%$ & $2.455 \pm 0.140$ & 2.887 & $25.66 \%$ \\
\hline 0.52 & 30 & $572.8 \pm 4.4$ & 564.8 & $1.41 \%$ & $1.416 \pm 0.058$ & 1.461 & $-0.97 \%$ \\
0.52 & 40 & $583.9 \pm 4.1$ & 577.5 & $1.11 \%$ & $1.571 \pm 0.105$ & 1.597 & $4.87 \%$ \\
0.52 & 50 & $593.0 \pm 3.9$ & 588.4 & $0.80 \%$ & $1.627 \pm 0.025$ & 1.725 & $8.37 \%$ \\
\hline 0.72 & 30 & $473.2 \pm 5.5$ & 463.2 & $2.17 \%$ & $0.854 \pm 0.023$ & 0.806 & $-9.31 \%$ \\
0.72 & 40 & $490.2 \pm 5.0$ & 481.6 & $1.80 \%$ & $0.956 \pm 0.060$ & 0.901 & $-8.40 \%$ \\
0.72 & 50 & $504.4 \pm 4.7$ & 496.3 & $1.64 \%$ & $1.050 \pm 0.060$ & 0.986 & $-6.14 \%$ \\
\hline 0.88 & 30 & $341.1 \pm 6.4$ & 335.6 & $1.65 \%$ & $0.466 \pm 0.015$ & 0.431 & $-2.96 \%$ \\
0.88 & 40 & $369.6 \pm 5.7$ & 364.2 & $1.51 \%$ & $0.546 \pm 0.036$ & 0.501 & $-7.57 \%$ \\
0.88 & 50 & $390.5 \pm 5.3$ & 384.9 & $1.48 \%$ & $0.605 \pm 0.014$ & 0.561 & $-7.71 \%$ \\
\hline 0.27 & 30 & $623.3 \pm 3.9$ & 615.1 & $1.33 \%$ & $1.844 \pm 0.068$ & 1.985 & $8.18 \%$ \\
0.27 & 40 & $633.6 \pm 3.8$ & 626.1 & $1.20 \%$ & $1.952 \pm 0.003$ & 2.161 & $8.55 \%$ \\
0.27 & 50 & $641.4 \pm 3.6$ & 635.8 & $0.88 \%$ & $2.064 \pm 0.114$ & 2.330 & $12.14 \%$ \\
\hline 0.52 & 30 & $547.3 \pm 4.9$ & 536.7 & $1.98 \%$ & $1.181 \pm 0.045$ & 1.193 & $0.67 \%$ \\
0.52 & 40 & $560.1 \pm 4.7$ & 552.0 & $1.48 \%$ & $1.289 \pm 0.020$ & 1.318 & $-1.33 \%$ \\
0.52 & 50 & $571.9 \pm 4.2$ & 564.8 & $1.26 \%$ & $1.458 \pm 0.015$ & 1.433 & $-1.45 \%$ \\
\hline 0.72 & 30 & $441.0 \pm 6.1$ & 430.0 & $2.56 \%$ & $0.708 \pm 0.015$ & 0.665 & $-6.14 \%$ \\
0.72 & 40 & $463.1 \pm 5.5$ & 452.9 & $2.25 \%$ & $0.822 \pm 0.047$ & 0.753 & $-4.52 \%$ \\
0.72 & 50 & $478.5 \pm 5.1$ & 470.6 & $1.69 \%$ & $0.873 \pm 0.007$ & 0.831 & $-3.08 \%$ \\
\hline 0.88 & 30 & $304.5 \pm 6.5$ & 299.2 & $1.77 \%$ & $0.387 \pm 0.017$ & 0.369 & $-4.17 \%$ \\
0.88 & 40 & $339.7 \pm 6.0$ & 333.8 & $1.77 \%$ & $0.442 \pm 0.021$ & 0.435 & $-9.08 \%$ \\
0.88 & 50 & $363.2 \pm 5.6$ & 358.1 & $1.42 \%$ & $0.512 \pm 0.025$ & 0.491 & $-9.36 \%$ \\
\hline
\end{tabular}


Table S5: Self-diffusion coefficients of methane in methane(1)/n-hexane(2) mixtures obtained with GROMOS Force Field

\begin{tabular}{|c|c|c|c|c|c|c|c|}
\hline \multirow{2}{*}{$\mathrm{T} / \mathrm{K}$} & \multirow{2}{*}{$x_{1}$} & \multirow{2}{*}{$\mathrm{P} / \mathrm{MPa}$} & \multicolumn{5}{|c|}{$D_{1} / 10^{-9} m^{2} s^{-1}$} \\
\hline & & & Exp & MD & $\mathbf{A A D}$ & $\mathbf{M D}+\mathbf{Y H}$ & AAD \\
\hline \multirow{12}{*}{303.2} & 0.27 & 30 & 10.00 & $10.67 \pm 0.18$ & 0.67 & $11.22 \pm 0.17$ & 1.22 \\
\hline & 0.27 & 40 & 9.10 & $9.89 \pm 0.04$ & 0.79 & $10.38 \pm 0.07$ & 1.28 \\
\hline & 0.27 & 50 & 8.90 & $9.18 \pm 0.21$ & 0.28 & $9.64 \pm 0.20$ & 0.74 \\
\hline & 0.52 & 30 & 16.50 & $14.84 \pm 0.14$ & 1.66 & $15.71 \pm 0.20$ & 0.79 \\
\hline & 0.52 & 40 & 15.00 & $13.74 \pm 0.10$ & 1.26 & $14.56 \pm 0.10$ & 0.44 \\
\hline & 0.52 & 50 & 14.00 & $12.78 \pm 0.02$ & 1.22 & $13.51 \pm 0.03$ & 0.49 \\
\hline & 0.72 & 30 & 26.00 & $22.73 \pm 0.15$ & 3.27 & $24.28 \pm 0.16$ & 1.72 \\
\hline & 0.72 & 40 & 23.00 & $20.32 \pm 0.03$ & 2.68 & $21.62 \pm 0.03$ & 1.38 \\
\hline & 0.72 & 50 & 21.00 & $18.37 \pm 0.07$ & 2.63 & $19.59 \pm 0.06$ & 1.41 \\
\hline & 0.88 & 30 & 41.00 & $37.62 \pm 0.13$ & 3.38 & $40.48 \pm 0.02$ & 0.52 \\
\hline & 0.88 & 40 & 34.00 & $31.54 \pm 0.15$ & 2.46 & $34.04 \pm 0.24$ & 0.17 \\
\hline & 0.88 & 50 & 30.00 & $27.70 \pm 0.03$ & 2.30 & $29.96 \pm 0.06$ & 0.06 \\
\hline \multirow{12}{*}{333.1} & 0.27 & 30 & 13.60 & $13.52 \pm 0.22$ & 0.20 & $14.22 \pm 0.20$ & 0.62 \\
\hline & 0.27 & 40 & 12.40 & $12.60 \pm 0.04$ & 0.20 & $13.20 \pm 0.02$ & 0.80 \\
\hline & 0.27 & 50 & 11.00 & $11.74 \pm 0.10$ & 0.74 & $12.31 \pm 0.10$ & 1.31 \\
\hline & 0.52 & 30 & 22.00 & $19.51 \pm 0.07$ & 2.49 & $20.64 \pm 0.07$ & 1.36 \\
\hline & 0.52 & 40 & 19.50 & $17.55 \pm 0.05$ & 1.95 & $18.56 \pm 0.06$ & 0.94 \\
\hline & 0.52 & 50 & 17.90 & $16.43 \pm 0.09$ & 1.47 & $17.38 \pm 0.10$ & 0.52 \\
\hline & 0.72 & 30 & 34.00 & $30.10 \pm 0.08$ & 3.90 & $32.13 \pm 0.09$ & 1.87 \\
\hline & 0.72 & 40 & 29.00 & $26.27 \pm 0.12$ & 2.73 & $28.04 \pm 0.10$ & 0.96 \\
\hline & 0.72 & 50 & 26.00 & $23.58 \pm 0.12$ & 2.42 & $25.22 \pm 0.13$ & 0.78 \\
\hline & 0.88 & 30 & 52.00 & $49.66 \pm 0.33$ & 2.34 & $53.35 \pm 0.26$ & 1.35 \\
\hline & 0.88 & 40 & 40.00 & $40.59 \pm 0.07$ & 0.59 & $43.71 \pm 0.08$ & 3.71 \\
\hline & 0.88 & 50 & 36.00 & $34.96 \pm 0.20$ & 1.04 & $37.70 \pm 0.31$ & 1.70 \\
\hline
\end{tabular}


Table S6: Self-diffusion coefficients of methane in methane(1)/n-hexane(2) mixtures obtained with NERD Force Field

\begin{tabular}{|c|c|c|c|c|c|c|c|}
\hline \multirow{2}{*}{$\mathbf{T} / \mathbf{K}$} & \multirow{2}{*}{$x_{1}$} & \multirow{2}{*}{$\mathrm{P} / \mathrm{MPa}$} & \multicolumn{5}{|c|}{$D_{1} / 10^{-9} m^{2} s^{-1}$} \\
\hline & & & $\operatorname{Exp}$ & MD & AAD & $\mathbf{M D}+\mathbf{Y H}$ & AAD \\
\hline \multirow{12}{*}{303.2} & 0.27 & 30 & 10.00 & $11.51 \pm 0.05$ & 1.51 & $12.11 \pm 0.06$ & 2.11 \\
\hline & 0.27 & 40 & 9.10 & $10.87 \pm 0.14$ & 1.77 & $11.45 \pm 0.14$ & 2.35 \\
\hline & 0.27 & 50 & 8.90 & $9.98 \pm 0.06$ & 1.08 & $10.51 \pm 0.06$ & 1.61 \\
\hline & 0.52 & 30 & 16.50 & $15.93 \pm 0.03$ & 0.57 & $16.89 \pm 0.01$ & 0.39 \\
\hline & 0.52 & 40 & 15.00 & $14.80 \pm 0.12$ & 0.20 & $15.66 \pm 0.10$ & 0.66 \\
\hline & 0.52 & 50 & 14.00 & $13.67 \pm 0.07$ & 0.33 & $14.47 \pm 0.09$ & 0.47 \\
\hline & 0.72 & 30 & 26.00 & $23.48 \pm 0.11$ & 2.52 & $25.01 \pm 0.11$ & 0.99 \\
\hline & 0.72 & 40 & 23.00 & $21.05 \pm 0.02$ & 1.95 & $22.46 \pm 0.05$ & 0.54 \\
\hline & 0.72 & 50 & 21.00 & $19.05 \pm 0.06$ & 1.95 & $20.37 \pm 0.05$ & 0.63 \\
\hline & 0.88 & 30 & 41.00 & $37.17 \pm 0.27$ & 3.83 & $40.19 \pm 0.21$ & 0.81 \\
\hline & 0.88 & 40 & 34.00 & $31.69 \pm 0.13$ & 2.31 & $34.09 \pm 0.04$ & 0.09 \\
\hline & 0.88 & 50 & 30.00 & $27.94 \pm 0.05$ & 2.06 & $30.25 \pm 0.06$ & 0.25 \\
\hline \multirow{12}{*}{333.1} & 0.27 & 30 & 13.60 & $15.00 \pm 0.09$ & 1.40 & $15.74 \pm 0.08$ & 2.14 \\
\hline & 0.27 & 40 & 12.40 & $13.89 \pm 0.02$ & 1.49 & $14.60 \pm 0.04$ & 2.20 \\
\hline & 0.27 & 50 & 11.00 & $12.86 \pm 0.09$ & 1.86 & $13.53 \pm 0.09$ & 2.53 \\
\hline & 0.52 & 30 & 22.00 & $20.69 \pm 0.36$ & 1.31 & $21.91 \pm 0.35$ & 0.30 \\
\hline & 0.52 & 40 & 19.50 & $18.73 \pm 0.11$ & 0.77 & $19.80 \pm 0.11$ & 0.30 \\
\hline & 0.52 & 50 & 17.90 & $17.38 \pm 0.05$ & 0.52 & $18.43 \pm 0.06$ & 0.53 \\
\hline & 0.72 & 30 & 34.00 & $30.70 \pm 0.18$ & 3.30 & $32.74 \pm 0.16$ & 1.26 \\
\hline & 0.72 & 40 & 29.00 & $27.01 \pm 0.07$ & 1.99 & $28.85 \pm 0.06$ & 0.15 \\
\hline & 0.72 & 50 & 26.00 & $24.27 \pm 0.03$ & 1.73 & $25.92 \pm 0.06$ & 0.08 \\
\hline & 0.88 & 30 & 52.00 & $49.03 \pm 0.38$ & 2.97 & $52.82 \pm 0.23$ & 0.82 \\
\hline & 0.88 & 40 & 40.00 & $40.49 \pm 0.23$ & 0.49 & $43.70 \pm 0.15$ & 3.70 \\
\hline & 0.88 & 50 & 36.00 & $35.05 \pm 0.17$ & 0.95 & $37.81 \pm 0.14$ & 1.81 \\
\hline
\end{tabular}


Table S7: Self-diffusion coefficients of methane in methane(1)/n-hexane(2) mixtures obtained with OPLS-AA Force Field

\begin{tabular}{|c|c|c|c|c|c|c|c|}
\hline \multirow{2}{*}{$\mathbf{T} / \mathbf{K}$} & \multirow{2}{*}{$x_{1}$} & \multirow{2}{*}{$\mathrm{P} / \mathrm{MPa}$} & \multicolumn{5}{|c|}{$D_{1} / 10^{-9} m^{2} s^{-1}$} \\
\hline & & & Exp & MD & AAD & $\mathbf{M D}+\mathbf{Y H}$ & AAD \\
\hline \multirow{12}{*}{303.2} & 0.27 & 30 & 10.00 & $9.04 \pm 0.05$ & 0.96 & $9.50 \pm 0.08$ & 0.50 \\
\hline & 0.27 & 40 & 9.10 & $8.36 \pm 0.05$ & 0.74 & $8.78 \pm 0.05$ & 0.32 \\
\hline & 0.27 & 50 & 8.90 & $7.81 \pm 0.08$ & 1.09 & $8.19 \pm 0.09$ & 0.71 \\
\hline & 0.52 & 30 & 16.50 & $13.91 \pm 0.04$ & 2.59 & $14.72 \pm 0.05$ & 1.78 \\
\hline & 0.52 & 40 & 15.00 & $12.70 \pm 0.03$ & 2.30 & $13.40 \pm 0.02$ & 1.60 \\
\hline & 0.52 & 50 & 14.00 & $11.67 \pm 0.12$ & 2.33 & $12.32 \pm 0.14$ & 1.68 \\
\hline & 0.72 & 30 & 26.00 & $22.31 \pm 0.06$ & 3.69 & $23.78 \pm 0.11$ & 2.22 \\
\hline & 0.72 & 40 & 23.00 & $19.81 \pm 0.15$ & 3.19 & $21.12 \pm 0.12$ & 1.88 \\
\hline & 0.72 & 50 & 21.00 & $18.02 \pm 0.10$ & 2.98 & $19.23 \pm 0.19$ & 1.77 \\
\hline & 0.88 & 30 & 41.00 & $37.60 \pm 0.18$ & 3.40 & $40.55 \pm 0.19$ & 0.45 \\
\hline & 0.88 & 40 & 34.00 & $32.07 \pm 0.12$ & 1.93 & $34.61 \pm 0.08$ & 0.61 \\
\hline & 0.88 & 50 & 30.00 & $28.27 \pm 0.11$ & 1.73 & $30.59 \pm 0.13$ & 0.59 \\
\hline \multirow{12}{*}{333.1} & 0.27 & 30 & 13.60 & $12.38 \pm 0.12$ & 1.22 & $12.98 \pm 0.13$ & 0.62 \\
\hline & 0.27 & 40 & 12.40 & $11.38 \pm 0.11$ & 1.02 & $11.93 \pm 0.11$ & 0.47 \\
\hline & 0.27 & 50 & 11.00 & $10.55 \pm 0.07$ & 0.45 & $11.08 \pm 0.08$ & 0.08 \\
\hline & 0.52 & 30 & 22.00 & $18.62 \pm 0.11$ & 3.38 & $19.65 \pm 0.13$ & 2.35 \\
\hline & 0.52 & 40 & 19.50 & $16.77 \pm 0.03$ & 2.73 & $17.76 \pm 0.04$ & 1.74 \\
\hline & 0.52 & 50 & 17.90 & $15.42 \pm 0.11$ & 2.48 & $16.29 \pm 0.15$ & 1.61 \\
\hline & 0.72 & 30 & 34.00 & $29.65 \pm 0.01$ & 4.35 & $31.60 \pm 0.12$ & 2.40 \\
\hline & 0.72 & 40 & 29.00 & $25.84 \pm 0.13$ & 3.16 & $27.55 \pm 0.15$ & 1.45 \\
\hline & 0.72 & 50 & 26.00 & $23.26 \pm 0.11$ & 2.74 & $24.85 \pm 0.15$ & 1.15 \\
\hline & 0.88 & 30 & 52.00 & $49.98 \pm 0.10$ & 2.02 & $53.77 \pm 0.33$ & 1.77 \\
\hline & 0.88 & 40 & 40.00 & $41.28 \pm 0.06$ & 1.28 & $44.52 \pm 0.06$ & 4.52 \\
\hline & 0.88 & 50 & 36.00 & $35.98 \pm 0.07$ & 0.05 & $38.80 \pm 0.05$ & 2.80 \\
\hline
\end{tabular}


Table S8: Self-diffusion coefficients of methane in methane(1)/n-hexane(2) mixtures obtained with OPLS-UA Force Field

\begin{tabular}{|c|c|c|c|c|c|c|c|}
\hline \multirow{2}{*}{$\mathbf{T} / \mathbf{K}$} & \multirow{2}{*}{$x_{1}$} & \multirow{2}{*}{$\mathrm{P} / \mathrm{MPa}$} & \multicolumn{5}{|c|}{$D_{1} / 10^{-9} m^{2} s^{-1}$} \\
\hline & & & $\operatorname{Exp}$ & MD & AAD & $\mathbf{M D}+\mathbf{Y H}$ & AAD \\
\hline \multirow{12}{*}{303.2} & 0.27 & 30 & 10.00 & $10.04 \pm 0.13$ & 0.11 & $10.57 \pm 0.14$ & 0.57 \\
\hline & 0.27 & 40 & 9.10 & $9.51 \pm 0.10$ & 0.41 & $10.01 \pm 0.13$ & 0.91 \\
\hline & 0.27 & 50 & 8.90 & $8.95 \pm 0.09$ & 0.07 & $9.42 \pm 0.07$ & 0.52 \\
\hline & 0.52 & 30 & 16.50 & $13.30 \pm 0.09$ & 0.30 & $13.96 \pm 0.11$ & 0.36 \\
\hline & 0.52 & 40 & 15.00 & $12.32 \pm 0.05$ & 0.08 & $12.95 \pm 0.05$ & 0.55 \\
\hline & 0.52 & 50 & 14.00 & $11.63 \pm 0.06$ & 0.63 & $12.24 \pm 0.06$ & 1.24 \\
\hline & 0.72 & 30 & 26.00 & $14.25 \pm 0.10$ & 2.25 & $15.10 \pm 0.08$ & 1.40 \\
\hline & 0.72 & 40 & 23.00 & $13.36 \pm 0.13$ & 1.64 & $14.14 \pm 0.16$ & 0.86 \\
\hline & 0.72 & 50 & 21.00 & $12.46 \pm 0.05$ & 1.54 & $13.20 \pm 0.04$ & 0.80 \\
\hline & 0.88 & 30 & 41.00 & $18.55 \pm 0.18$ & 3.45 & $19.65 \pm 0.16$ & 2.35 \\
\hline & 0.88 & 40 & 34.00 & $17.00 \pm 0.10$ & 2.50 & $18.02 \pm 0.11$ & 1.48 \\
\hline & 0.88 & 50 & 30.00 & $15.85 \pm 0.13$ & 2.05 & $16.75 \pm 0.14$ & 1.15 \\
\hline \multirow{12}{*}{333.1} & 0.27 & 30 & 13.60 & $21.77 \pm 0.11$ & 4.23 & $23.24 \pm 0.14$ & 2.76 \\
\hline & 0.27 & 40 & 12.40 & $19.68 \pm 0.05$ & 3.32 & $21.02 \pm 0.06$ & 1.98 \\
\hline & 0.27 & 50 & 11.00 & $17.94 \pm 0.09$ & 3.06 & $19.16 \pm 0.13$ & 1.84 \\
\hline & 0.52 & 30 & 22.00 & $28.64 \pm 0.06$ & 5.36 & $30.55 \pm 0.02$ & 3.45 \\
\hline & 0.52 & 40 & 19.50 & $25.14 \pm 0.06$ & 3.86 & $26.81 \pm 0.06$ & 2.19 \\
\hline & 0.52 & 50 & 17.90 & $22.76 \pm 0.02$ & 3.24 & $24.35 \pm 0.03$ & 1.65 \\
\hline & 0.72 & 30 & 34.00 & $36.29 \pm 0.14$ & 4.71 & $39.04 \pm 0.09$ & 1.96 \\
\hline & 0.72 & 40 & 29.00 & $30.80 \pm 0.14$ & 3.20 & $33.21 \pm 0.30$ & 0.79 \\
\hline & 0.72 & 50 & 26.00 & $27.10 \pm 0.28$ & 2.90 & $29.31 \pm 0.31$ & 0.69 \\
\hline & 0.88 & 30 & 52.00 & $47.86 \pm 0.14$ & 4.14 & $51.36 \pm 0.18$ & 0.64 \\
\hline & 0.88 & 40 & 40.00 & $39.28 \pm 0.04$ & 0.72 & $42.46 \pm 0.19$ & 2.46 \\
\hline & 0.88 & 50 & 36.00 & $34.45 \pm 0.09$ & 1.55 & $37.26 \pm 0.20$ & 1.26 \\
\hline
\end{tabular}


Table S9: Self-diffusion coefficients of n-hexane in methane(1)/n-hexane(2) mixtures obtained with GROMOS Force Field

\begin{tabular}{|c|c|c|c|c|c|c|c|}
\hline \multirow{2}{*}{$\mathrm{T} / \mathrm{K}$} & \multirow{2}{*}{$x_{1}$} & \multirow{2}{*}{$\mathrm{P} / \mathrm{MPa}$} & \multicolumn{5}{|c|}{$D_{2} / 10^{-9} m^{2} s^{-1}$} \\
\hline & & & Exp & MD & AAD & $\mathbf{M D}+\mathbf{Y H}$ & AAD \\
\hline \multirow{12}{*}{303.2} & 0.27 & 30 & 4.70 & $5.12 \pm 0.02$ & 0.42 & $5.67 \pm 0.03$ & 0.97 \\
\hline & 0.27 & 40 & 4.30 & $4.77 \pm 0.03$ & 0.47 & $5.27 \pm 0.00$ & 0.97 \\
\hline & 0.27 & 50 & 4.10 & $4.41 \pm 0.01$ & 0.31 & $4.87 \pm 0.02$ & 0.77 \\
\hline & 0.52 & 30 & 7.70 & $7.17 \pm 0.05$ & 0.53 & $8.04 \pm 0.02$ & 0.34 \\
\hline & 0.52 & 40 & 7.30 & $6.55 \pm 0.12$ & 0.75 & $7.36 \pm 0.13$ & 0.10 \\
\hline & 0.52 & 50 & 6.60 & $6.18 \pm 0.03$ & 0.42 & $6.92 \pm 0.04$ & 0.32 \\
\hline & 0.72 & 30 & 12.20 & $10.69 \pm 0.15$ & 1.51 & $12.25 \pm 0.09$ & 0.07 \\
\hline & 0.72 & 40 & 11.10 & $9.72 \pm 0.11$ & 1.38 & $11.02 \pm 0.11$ & 0.09 \\
\hline & 0.72 & 50 & 9.90 & $8.86 \pm 0.09$ & 1.04 & $10.07 \pm 0.08$ & 0.17 \\
\hline & 0.88 & 30 & 18.50 & $17.02 \pm 0.13$ & 1.48 & $19.89 \pm 0.20$ & 1.39 \\
\hline & 0.88 & 40 & 16.60 & $14.67 \pm 0.16$ & 1.93 & $17.17 \pm 0.26$ & 0.57 \\
\hline & 0.88 & 50 & 15.60 & $13.52 \pm 0.21$ & 2.08 & $15.79 \pm 0.21$ & 0.19 \\
\hline \multirow{12}{*}{333.1} & 0.27 & 30 & 6.30 & $6.53 \pm 0.02$ & 0.23 & $7.24 \pm 0.03$ & 0.94 \\
\hline & 0.27 & 40 & 5.70 & $6.07 \pm 0.08$ & 0.37 & $6.68 \pm 0.10$ & 0.98 \\
\hline & 0.27 & 50 & 5.30 & $5.72 \pm 0.02$ & 0.42 & $6.30 \pm 0.03$ & 1.00 \\
\hline & 0.52 & 30 & 10.30 & $9.35 \pm 0.10$ & 0.95 & $10.48 \pm 0.07$ & 0.18 \\
\hline & 0.52 & 40 & 9.40 & $8.54 \pm 0.03$ & 0.86 & $9.54 \pm 0.04$ & 0.14 \\
\hline & 0.52 & 50 & 8.40 & $7.96 \pm 0.02$ & 0.44 & $8.91 \pm 0.04$ & 0.51 \\
\hline & 0.72 & 30 & 16.40 & $14.28 \pm 0.15$ & 2.12 & $16.31 \pm 0.15$ & 0.10 \\
\hline & 0.72 & 40 & 14.20 & $12.52 \pm 0.09$ & 1.68 & $14.29 \pm 0.08$ & 0.09 \\
\hline & 0.72 & 50 & 13.20 & $11.40 \pm 0.09$ & 1.80 & $13.03 \pm 0.07$ & 0.17 \\
\hline & 0.88 & 30 & 23.00 & $22.99 \pm 0.28$ & 0.20 & $26.68 \pm 0.31$ & 3.68 \\
\hline & 0.88 & 40 & 19.20 & $19.16 \pm 0.12$ & 0.09 & $22.28 \pm 0.16$ & 3.08 \\
\hline & 0.88 & 50 & 16.40 & $16.76 \pm 0.54$ & 0.50 & $19.51 \pm 0.50$ & 3.11 \\
\hline
\end{tabular}


Table S10: Self-diffusion coefficients of n-hexane in methane(1)/n-hexane(2) mixtures obtained with NERD Force Field

\begin{tabular}{|c|c|c|c|c|c|c|c|}
\hline \multirow{2}{*}{$\mathrm{T} / \mathrm{K}$} & \multirow{2}{*}{$x_{1}$} & \multirow{2}{*}{$\mathrm{P} / \mathrm{MPa}$} & \multicolumn{5}{|c|}{$D_{2} / 10^{-9} m^{2} s^{-1}$} \\
\hline & & & Exp & MD & $\mathbf{A A D}$ & $\mathbf{M D}+\mathbf{Y H}$ & AAD \\
\hline \multirow{12}{*}{303.2} & 0.27 & 30 & 4.70 & $5.76 \pm 0.02$ & 1.06 & $6.36 \pm 0.01$ & 1.66 \\
\hline & 0.27 & 40 & 4.30 & $5.34 \pm 0.02$ & 1.04 & $5.92 \pm 0.02$ & 1.62 \\
\hline & 0.27 & 50 & 4.10 & $4.97 \pm 0.02$ & 0.87 & $5.50 \pm 0.03$ & 1.40 \\
\hline & 0.52 & 30 & 7.70 & $7.89 \pm 0.14$ & 0.19 & $8.85 \pm 0.11$ & 1.15 \\
\hline & 0.52 & 40 & 7.30 & $7.28 \pm 0.03$ & 0.02 & $8.14 \pm 0.00$ & 0.84 \\
\hline & 0.52 & 50 & 6.60 & $6.86 \pm 0.08$ & 0.26 & $7.66 \pm 0.06$ & 1.06 \\
\hline & 0.72 & 30 & 12.20 & $11.49 \pm 0.19$ & 0.71 & $13.02 \pm 0.18$ & 0.82 \\
\hline & 0.72 & 40 & 11.10 & $10.44 \pm 0.15$ & 0.66 & $11.86 \pm 0.19$ & 0.76 \\
\hline & 0.72 & 50 & 9.90 & $9.48 \pm 0.06$ & 0.42 & $10.80 \pm 0.08$ & 0.90 \\
\hline & 0.88 & 30 & 18.50 & $18.30 \pm 0.20$ & 0.22 & $21.32 \pm 0.25$ & 2.82 \\
\hline & 0.88 & 40 & 16.60 & $15.68 \pm 0.15$ & 0.92 & $18.08 \pm 0.07$ & 1.48 \\
\hline & 0.88 & 50 & 15.60 & $14.05 \pm 0.00$ & 1.55 & $16.36 \pm 0.02$ & 0.76 \\
\hline \multirow{12}{*}{333.1} & 0.27 & 30 & 6.30 & $7.45 \pm 0.01$ & 1.15 & $8.19 \pm 0.01$ & 1.89 \\
\hline & 0.27 & 40 & 5.70 & $6.95 \pm 0.09$ & 1.25 & $7.66 \pm 0.06$ & 1.96 \\
\hline & 0.27 & 50 & 5.30 & $6.49 \pm 0.05$ & 1.19 & $7.16 \pm 0.05$ & 1.86 \\
\hline & 0.52 & 30 & 10.30 & $10.28 \pm 0.14$ & 0.11 & $11.50 \pm 0.12$ & 1.20 \\
\hline & 0.52 & 40 & 9.40 & $9.42 \pm 0.09$ & 0.07 & $10.49 \pm 0.08$ & 1.09 \\
\hline & 0.52 & 50 & 8.40 & $8.73 \pm 0.07$ & 0.33 & $9.78 \pm 0.07$ & 1.38 \\
\hline & 0.72 & 30 & 16.40 & $15.03 \pm 0.24$ & 1.37 & $17.07 \pm 0.19$ & 0.67 \\
\hline & 0.72 & 40 & 14.20 & $13.39 \pm 0.15$ & 0.81 & $15.23 \pm 0.12$ & 1.03 \\
\hline & 0.72 & 50 & 13.20 & $12.14 \pm 0.14$ & 1.06 & $13.79 \pm 0.10$ & 0.59 \\
\hline & 0.88 & 30 & 23.00 & $23.89 \pm 0.25$ & 0.89 & $27.68 \pm 0.23$ & 4.68 \\
\hline & 0.88 & 40 & 19.20 & $20.16 \pm 0.11$ & 0.96 & $23.38 \pm 0.18$ & 4.18 \\
\hline & 0.88 & 50 & 16.40 & $17.44 \pm 0.12$ & 1.04 & $20.20 \pm 0.14$ & 3.80 \\
\hline
\end{tabular}


Table S11: Self-diffusion coefficients of n-hexane in methane(1)/n-hexane(2) mixtures obtained with OPLS-AA Force Field

\begin{tabular}{|c|c|c|c|c|c|c|c|}
\hline \multirow{2}{*}{$\mathbf{T} / \mathbf{K}$} & \multirow{2}{*}{$x_{1}$} & \multirow{2}{*}{$\mathrm{P} / \mathrm{MPa}$} & \multicolumn{5}{|c|}{$D_{2} / 10^{-9} m^{2} s^{-1}$} \\
\hline & & & Exp & MD & AAD & $\mathbf{M D}+\mathbf{Y H}$ & AAD \\
\hline \multirow{12}{*}{303.2} & 0.27 & 30 & 4.70 & $3.81 \pm 0.01$ & 0.89 & $4.27 \pm 0.03$ & 0.43 \\
\hline & 0.27 & 40 & 4.30 & $3.54 \pm 0.04$ & 0.76 & $3.96 \pm 0.04$ & 0.34 \\
\hline & 0.27 & 50 & 4.10 & $3.29 \pm 0.02$ & 0.81 & $3.67 \pm 0.01$ & 0.43 \\
\hline & 0.52 & 30 & 7.70 & $5.91 \pm 0.01$ & 1.79 & $6.72 \pm 0.04$ & 0.98 \\
\hline & 0.52 & 40 & 7.30 & $5.43 \pm 0.01$ & 1.87 & $6.14 \pm 0.03$ & 1.16 \\
\hline & 0.52 & 50 & 6.60 & $5.02 \pm 0.07$ & 1.58 & $5.67 \pm 0.08$ & 0.93 \\
\hline & 0.72 & 30 & 12.20 & $9.62 \pm 0.11$ & 2.58 & $11.09 \pm 0.14$ & 1.11 \\
\hline & 0.72 & 40 & 11.10 & $8.56 \pm 0.02$ & 2.54 & $9.87 \pm 0.05$ & 1.23 \\
\hline & 0.72 & 50 & 9.90 & $7.87 \pm 0.03$ & 2.03 & $9.08 \pm 0.14$ & 0.82 \\
\hline & 0.88 & 30 & 18.50 & $16.31 \pm 0.17$ & 2.19 & $19.26 \pm 0.19$ & 0.76 \\
\hline & 0.88 & 40 & 16.60 & $14.37 \pm 0.32$ & 2.23 & $16.91 \pm 0.37$ & 0.33 \\
\hline & 0.88 & 50 & 15.60 & $12.83 \pm 0.19$ & 2.77 & $15.14 \pm 0.18$ & 0.46 \\
\hline \multirow{12}{*}{333.1} & 0.27 & 30 & 6.30 & $5.32 \pm 0.06$ & 0.98 & $5.92 \pm 0.07$ & 0.38 \\
\hline & 0.27 & 40 & 5.70 & $4.89 \pm 0.02$ & 0.81 & $5.44 \pm 0.02$ & 0.26 \\
\hline & 0.27 & 50 & 5.30 & $4.50 \pm 0.01$ & 0.80 & $5.03 \pm 0.01$ & 0.27 \\
\hline & 0.52 & 30 & 10.30 & $8.06 \pm 0.09$ & 2.24 & $9.09 \pm 0.12$ & 1.21 \\
\hline & 0.52 & 40 & 9.40 & $7.36 \pm 0.04$ & 2.04 & $8.35 \pm 0.02$ & 1.05 \\
\hline & 0.52 & 50 & 8.40 & $6.68 \pm 0.04$ & 1.72 & $7.55 \pm 0.03$ & 0.85 \\
\hline & 0.72 & 30 & 16.40 & $12.81 \pm 0.05$ & 3.59 & $14.75 \pm 0.14$ & 1.65 \\
\hline & 0.72 & 40 & 14.20 & $11.49 \pm 0.08$ & 2.71 & $13.20 \pm 0.11$ & 1.00 \\
\hline & 0.72 & 50 & 13.20 & $10.29 \pm 0.06$ & 2.91 & $11.88 \pm 0.04$ & 1.32 \\
\hline & 0.88 & 30 & 23.00 & $21.75 \pm 0.31$ & 1.25 & $25.54 \pm 0.18$ & 2.54 \\
\hline & 0.88 & 40 & 19.20 & $18.65 \pm 0.25$ & 0.55 & $21.90 \pm 0.28$ & 2.70 \\
\hline & 0.88 & 50 & 16.40 & $16.37 \pm 0.13$ & 0.09 & $19.20 \pm 0.23$ & 2.80 \\
\hline
\end{tabular}


Table S12: Self-diffusion coefficients of n-hexane in methane(1)/n-hexane(2) mixtures obtained with OPLS-UA Force Field

\begin{tabular}{|c|c|c|c|c|c|c|c|}
\hline \multirow{2}{*}{$\mathbf{T} / \mathbf{K}$} & \multirow{2}{*}{$x_{1}$} & \multirow{2}{*}{$\mathrm{P} / \mathrm{MPa}$} & \multicolumn{5}{|c|}{$D_{2} / 10^{-9} m^{2} s^{-1}$} \\
\hline & & & Exp & MD & AAD & $\mathbf{M D}+\mathbf{Y H}$ & AAD \\
\hline \multirow{12}{*}{303.2} & 0.27 & 30 & 4.70 & $5.02 \pm 0.03$ & 0.32 & $5.55 \pm 0.05$ & 0.85 \\
\hline & 0.27 & 40 & 4.30 & $4.74 \pm 0.02$ & 0.44 & $5.24 \pm 0.04$ & 0.94 \\
\hline & 0.27 & 50 & 4.10 & $4.43 \pm 0.03$ & 0.33 & $4.90 \pm 0.05$ & 0.80 \\
\hline & 0.52 & 30 & 7.70 & $6.58 \pm 0.01$ & 0.28 & $7.24 \pm 0.02$ & 0.94 \\
\hline & 0.52 & 40 & 7.30 & $6.08 \pm 0.06$ & 0.38 & $6.71 \pm 0.06$ & 1.01 \\
\hline & 0.52 & 50 & 6.60 & $5.76 \pm 0.02$ & 0.46 & $6.37 \pm 0.03$ & 1.07 \\
\hline & 0.72 & 30 & 12.20 & $6.97 \pm 0.05$ & 0.73 & $7.82 \pm 0.08$ & 0.12 \\
\hline & 0.72 & 40 & 11.10 & $6.54 \pm 0.03$ & 0.76 & $7.32 \pm 0.03$ & 0.03 \\
\hline & 0.72 & 50 & 9.90 & $6.15 \pm 0.01$ & 0.45 & $6.90 \pm 0.02$ & 0.30 \\
\hline & 0.88 & 30 & 18.50 & $9.19 \pm 0.13$ & 1.11 & $10.29 \pm 0.16$ & 0.12 \\
\hline & 0.88 & 40 & 16.60 & $8.45 \pm 0.11$ & 0.95 & $9.47 \pm 0.12$ & 0.11 \\
\hline & 0.88 & 50 & 15.60 & $7.87 \pm 0.07$ & 0.53 & $8.77 \pm 0.07$ & 0.37 \\
\hline \multirow{12}{*}{333.1} & 0.27 & 30 & 6.30 & $10.51 \pm 0.18$ & 1.69 & $11.98 \pm 0.18$ & 0.22 \\
\hline & 0.27 & 40 & 5.70 & $9.50 \pm 0.07$ & 1.60 & $10.83 \pm 0.05$ & 0.27 \\
\hline & 0.27 & 50 & 5.30 & $8.72 \pm 0.05$ & 1.18 & $9.94 \pm 0.11$ & 0.10 \\
\hline & 0.52 & 30 & 10.30 & $13.68 \pm 0.08$ & 2.72 & $15.60 \pm 0.08$ & 0.80 \\
\hline & 0.52 & 40 & 9.40 & $12.22 \pm 0.04$ & 1.98 & $13.90 \pm 0.10$ & 0.30 \\
\hline & 0.52 & 50 & 8.40 & $11.21 \pm 0.06$ & 1.99 & $12.80 \pm 0.07$ & 0.40 \\
\hline & 0.72 & 30 & 16.40 & $16.68 \pm 0.41$ & 1.82 & $19.43 \pm 0.32$ & 0.93 \\
\hline & 0.72 & 40 & 14.20 & $14.81 \pm 0.16$ & 1.79 & $17.23 \pm 0.26$ & 0.63 \\
\hline & 0.72 & 50 & 13.20 & $13.35 \pm 0.06$ & 2.25 & $15.56 \pm 0.02$ & 0.04 \\
\hline & 0.88 & 30 & 23.00 & $22.68 \pm 0.37$ & 0.38 & $26.18 \pm 0.23$ & 3.18 \\
\hline & 0.88 & 40 & 19.20 & $19.11 \pm 0.41$ & 0.27 & $22.29 \pm 0.26$ & 3.09 \\
\hline & 0.88 & 50 & 16.40 & $16.86 \pm 0.19$ & 0.46 & $19.67 \pm 0.16$ & 3.27 \\
\hline
\end{tabular}

